\title{
A General Synthesis of Iminosugars
}

\author{
Ciaran McDonnell, Linda Cronin, Julie L. O'Brien and Paul V. Murphy*
}

Centre for Synthesis and Chemical Biology, Chemistry Department, Conway Institute of Biomolecular and Biomedical Research, University College Dublin, Belfield, Dublin 4, Ireland

Email: Paul.V.Murphy@ucd.ie

\section{Index to Supporting Information}

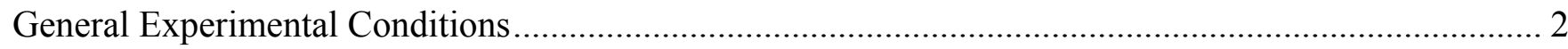

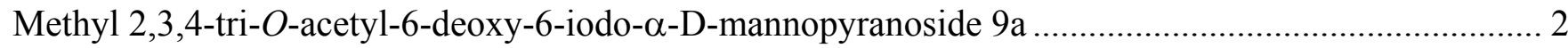

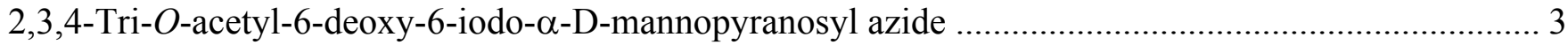

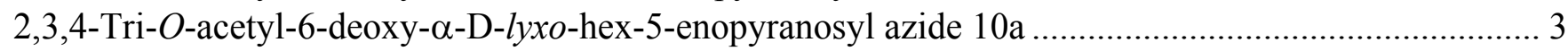

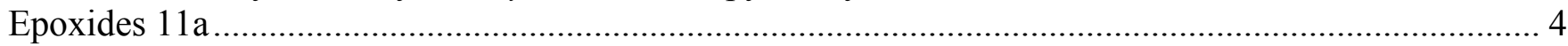

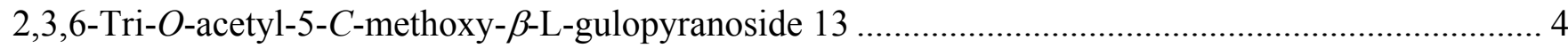

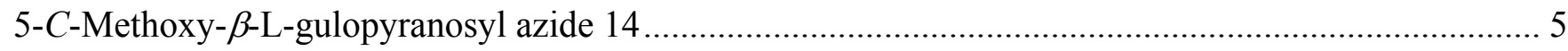

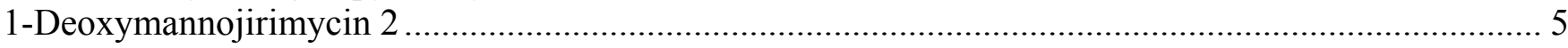

Methyl 2,3,4-tri- $O$-acetyl-6-deoxy-6-iodo- $\alpha$-D-glucopyranoside 9b ........................................................ 5

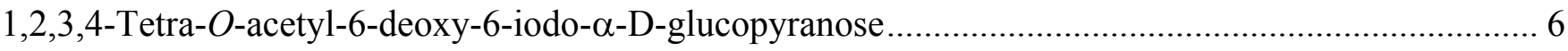

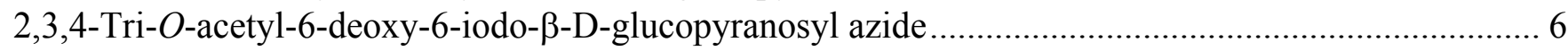

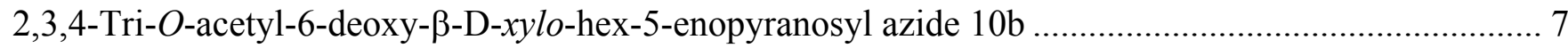

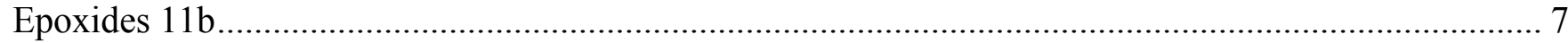

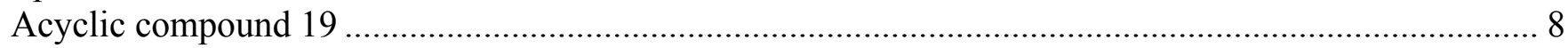

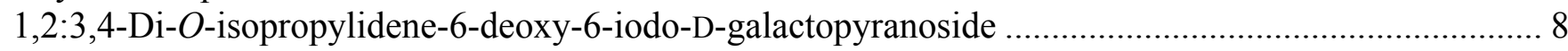

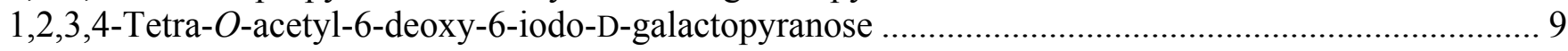

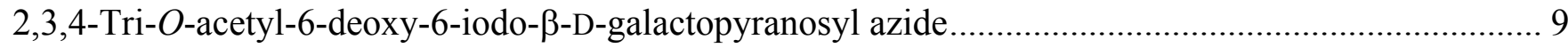

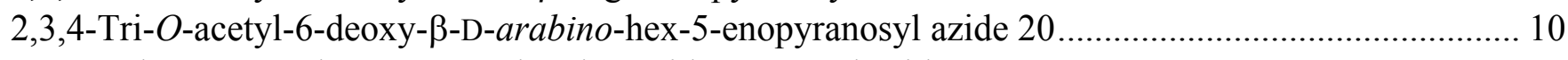

5-C-Methoxy- $\beta$-D-glucopyranosyl and $\alpha$-L-idopyranosyl azide ................................................. 10

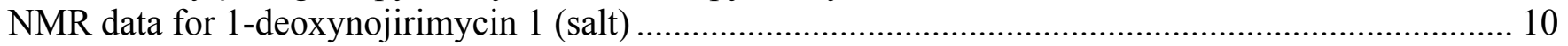

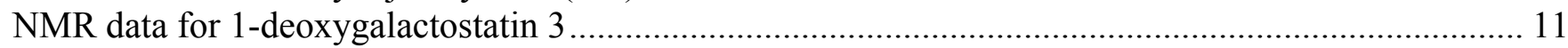

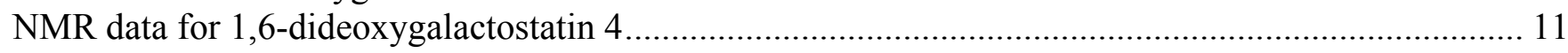

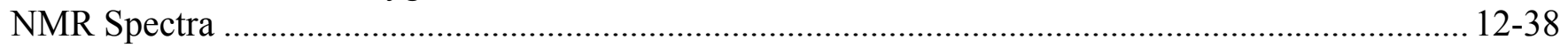




\section{General Experimental Conditions}

Optical rotations were determined at the sodium D line at $23{ }^{\circ} \mathrm{C}$. Chemical shifts in ${ }^{1} \mathrm{H}-\mathrm{NMR}$ spectra are reported relative to internal $\mathrm{Me}_{4} \mathrm{Si}$ in $\mathrm{CDCl}_{3}(\delta 0.0)$ or $\mathrm{HOD}$ for $\mathrm{D}_{2} \mathrm{O}(\delta 4.63)$ or $\mathrm{CD}_{2} \mathrm{HOD}(\delta 3.36)$ for ${ }^{1} \mathrm{H}$ and $\mathrm{CDCl}_{3}(\delta 77.0)$ or $\mathrm{CD}_{3} \mathrm{OD}(\delta 47.7)$ for ${ }^{13} \mathrm{C}$. ${ }^{1} \mathrm{H}$ NMR signals were assigned with the aid of COSY. ${ }^{13} \mathrm{C}-\mathrm{NMR}$ signals were assigned with the aid of DEPT. Coupling constants are reported in hertz. The IR spectra were recorded using either a thin film between $\mathrm{NaCl}$ plates or $\mathrm{KBr}$ discs, as specified. Thin layer chromatography (TLC) was performed on aluminium sheets precoated with silica gel $60\left(\mathrm{HF}_{254}\right.$, E. Merck) and spots visualized by UV and charring with $\mathrm{H}_{2} \mathrm{SO}_{4}-\mathrm{EtOH}(1: 20)$. Flash column chromatography was carried out with silica gel $60(0.040-0.630 \mathrm{~mm}$, E. Merck) and using a stepwise solvent polarity gradient (EtOAc-petroleum ether unless otherwise stated) correlated with TLC mobility. Chromatography solvents used were EtOAc and $\mathrm{MeOH}$ (Riedel-deHaen) and petroleum ether (b.p. $40-60^{\circ} \mathrm{C}, \mathrm{BDH}$ laboratory supplies). Toluene (Aldrich) and $\mathrm{CH}_{2} \mathrm{Cl}_{2}$ (Riedel-deHaen) were freshly distilled from calcium hydride.

\section{Methyl 2,3,4-tri-O-acetyl-6-deoxy-6-iodo- $\alpha$-D-mannopyranoside 9a}

To a solution of methyl $\alpha$-D-mannopyranoside $(9 \mathrm{~g}, 46.3 \mathrm{mmol})$ in toluene $(200 \mathrm{~mL})$ at $80{ }^{\circ} \mathrm{C}$ was added triphenylphosphine $(13.9 \mathrm{~g}, 53.3 \mathrm{mmol})$ and imidazole $(10.9 \mathrm{~g}, 160 \mathrm{mmol})$. The solution was stirred for $10 \mathrm{~min}$ and then iodine $(17.2 \mathrm{~g}, 66.7 \mathrm{mmol})$ was added portionwise over $0.5 \mathrm{~h}$. After a further $2 \mathrm{~h}$ heating the solution was cooled and the intermediate extracted into water $(3 \mathrm{x} 200 \mathrm{~mL})$. The combined water layers were then washed with toluene, the water removed in vacuo and the residue dissolved in acetic anhydride and pyridine $(1: 1,200 \mathrm{~mL})$. The solution was left to stand for $12 \mathrm{~h}$ after which time pyridine and acetic anhydride were removed in vacuo and xylene was evaporated from residue (2 x 50 $\mathrm{mL}$ ). The residue (a dark syrup) was reacted without further purification in the next step. A small portion of the material was purified further by chromatography to obtain an analytical sample of the title compound $^{1}$ and the remainder used in the next reaction without further purification; $[\alpha]_{\mathrm{D}}+42.4^{\mathrm{o}}(c) 2.5$, $\left.\mathrm{CHCl}_{3}\right) ;{ }^{1} \mathrm{H} \mathrm{NMR}\left(300 \mathrm{MHz}, \mathrm{CDCl}_{3}\right): \delta 5.31(\mathrm{dd}, 1 \mathrm{H}, J=3.5$ and 10.0), $5.22(\mathrm{dd}, 1 \mathrm{H}, J=1.5$ and 10.0), 5.11 (apparent t, $1 \mathrm{H}, J=9.5), 4.73(\mathrm{~d}, 1 \mathrm{H}, J=1.5), 3.80$ (apparent dt, $1 \mathrm{H}, J=2.5$ and 9.5) $3.48(\mathrm{~s}, 3 \mathrm{H}$, $\left.\mathrm{OCH}_{3}\right), 3.31(\mathrm{dd}, 1 \mathrm{H}, J=10.5$ and 2.5), $3.18(\mathrm{dd}, 1 \mathrm{H}, J=10.5$ and 9.5), 2.15, 2.07, 1.99 (each s, each $\left.\mathrm{CH}_{3}\right) ;{ }^{13} \mathrm{C}$ NMR $\left(125 \mathrm{MHz}, \mathrm{CDCl}_{3}\right): \delta 170.2,170.1,170.0$ (each s, each $\left.\mathrm{C}=\mathrm{O}\right), 98.8(\mathrm{~d}, \mathrm{C}-1), 70.4,69.9$,

\footnotetext{
${ }^{1}$ Garegg, P. J.; Samuelsson, B. J. Chem. Soc. Perkin Trans. 1 1980, 2866.
} 
69.0, (each d), 55.8 (q, $\left.\mathrm{OCH}_{3}\right), 21.1,21.0,20.9$ (each q, each $\left.\mathrm{CH}_{3}\right), 4.1(\mathrm{t}, \mathrm{C}-6)$; IR $(\mathrm{KBr}): 1748(\mathrm{C}=\mathrm{O})$, 1376, 1245, 1135, 1042, $972 \mathrm{~cm}^{-1}$. Anal. Calcd. for $\mathrm{C}_{13} \mathrm{H}_{19} \mathrm{O}_{8} \mathrm{I}: \mathrm{C}, 36.28$; H, 4.42; I, 29.54. Found: C, $36.24 ; \mathrm{H}, 4.48 ; \mathrm{I}, 29.62$.

\section{2,3,4-Tri-O-acetyl-6-deoxy-6-iodo- $\alpha$-D-mannopyranosyl azide}

The iodide 9a was dissolved in acetic anhydride:sulphuric acid (50:1, $250 \mathrm{~mL})$ and the mixture allowed to stir for $12 \mathrm{~h}$ at $\mathrm{rt}$. The solution was then concentrated in vacuo and the residue separated between EtOAc and water. The organic layer was washed several times with aq. $\mathrm{NaHCO}_{3}(5 \times 500 \mathrm{~mL})$, then water $(2 \times 500 \mathrm{~mL})$ and the removal of solvent in vacuo gave 1,2,3,4-tetra- $O$-acetyl-6-deoxy-6-iodo- $\alpha$ D-mannopyranose as a pale yellow oil which was used in the next reaction without further purification; $[\alpha]_{\mathrm{D}}+39.7^{\circ}\left(c\right.$ 1.5, $\left.\mathrm{CHCl}_{3}\right) ;{ }^{1} \mathrm{H} \mathrm{NMR}\left(300 \mathrm{MHz}, \mathrm{CDCl}_{3}\right): \delta 6.10(\mathrm{~d}, 1 \mathrm{H}, J=1.5), 5.34(\mathrm{dd}, 1 \mathrm{H}, J=3.5$ and 10.0), 5.24 (overlapping signals $2 \mathrm{H}$ ), 3.80 (apparent dt, $1 \mathrm{H}, J=2.5,7.0$ and 9.0), 3.32 (dd, 1H, $J=$ 11.0 and 2.5), $3.17\left(\mathrm{dd}, 1 \mathrm{H}, J=11.0\right.$ and 7.0), 2.17, 2.08, 2.01 (each s, 9H); ${ }^{13} \mathrm{C} \mathrm{NMR}(125 \mathrm{MHz}$, $\mathrm{CDCl}_{3}$ ): $\delta 170.1,169.9,169.7,168.1$ (each s, each $\left.\mathrm{C}=\mathrm{O}\right), 90.6(\mathrm{~d}, \mathrm{C}-1), 72.0,69.8,68.7,68.6$ (each d), 21.0, 20.9, 20.9, 20.7 (each q, each $\mathrm{CH}_{3}$ ), 3.7 (t, C-6); IR (KBr): 1748 (C=O), 1371, 1220, 1151, 1058 $\mathrm{cm}^{-1}$. CI-HRMS: Found 476.0422, required $476.0418\left(\mathrm{M}+\mathrm{NH}_{4}\right)^{+}$. To a solution of this intermediate in $\mathrm{CH}_{2} \mathrm{Cl}_{2}(250 \mathrm{~mL})$ under an inert atmosphere was added first $\mathrm{SnCl}_{4}(3 \mathrm{~mL}, 25 \mathrm{mmol})$, then TMS-N 3 (10 $\mathrm{mL}, 76 \mathrm{mmol}$ ). The resulting solution was stirred at room temp for $10 \mathrm{~min}$, then diluted with $\mathrm{CH}_{2} \mathrm{Cl}_{2}$ $(300 \mathrm{~mL})$, washed with aq. $\mathrm{NaHCO}_{3}(4 \times 500 \mathrm{~mL})$, water $(2$ x $500 \mathrm{~mL})$, dried over magnesium sulphate; the solvent was removed to give the title compound as a pale oil (quantitative yield) which was used in the next reaction without further purification; $[\alpha]_{\mathrm{D}}+86.5^{\circ}\left(c 1.1, \mathrm{CHCl}_{3}\right) ;{ }^{1} \mathrm{H} \mathrm{NMR}\left(300 \mathrm{MHz}, \mathrm{CDCl}_{3}\right)$ : $\delta 5.41(\mathrm{~d}, 1 \mathrm{H}, J=2.0), 5.24(\mathrm{dd}, 1 \mathrm{H}, J=3.5$ and 9.5), $5.16($ apparent t, $1 \mathrm{H}, J=9.5), 5.12(\mathrm{dd}, 1 \mathrm{H}, J=$ 2.0 and 3.5), $3.92(\mathrm{ddd}, 1 \mathrm{H}, J=3.0$ and 8.0), $3.35(\mathrm{dd}, 1 \mathrm{H}, J=11.0$ and 3.0), $3.18(\mathrm{dd}, 1 \mathrm{H}, J=11.0$ and 8.0), 2.16, 2.08, 1.99 (each s, 9H); ${ }^{13} \mathrm{C} \mathrm{NMR}\left(125 \mathrm{MHz}, \mathrm{CDCl}_{3}\right.$ ): $\delta 170.0,169.9,169.8$ (each s, each $\mathrm{C}=\mathrm{O}), 87.6$ (d, C-1), 72.1, 69.8, 69.5, 69.2 (each d), 21.0, 20.9, 20.8 (each q, each $\mathrm{CH}_{3}$ ), 3.5 (t, C-6); IR (thin film): $2121\left(\mathrm{~N}_{3}\right), 1753(\mathrm{C}=\mathrm{O}), 1372,1235,1126,1055,949 \mathrm{~cm}^{-1}$. CI-HRMS: Found 459.0372 , required $459.0377\left(\mathrm{M}+\mathrm{NH}_{4}\right)^{+}$.

\section{2,3,4-Tri- $O$-acetyl-6-deoxy- $\alpha$-D-lyxo-hex-5-enopyranosyl azide 10a}

2,3,4-Tri-O-acetyl-6-deoxy-6-iodo- $\alpha$-D-mannopyranosyl azide from the previous reaction and DBU $(14.4 \mathrm{~mL}, 96.4 \mathrm{mmol})$ in toluene $(150 \mathrm{~mL})$ was stirred and heated at reflux for $1.5 \mathrm{~h}$. The mixture was 
then washed with water, concentrated and the residue chromatographed to give 10a as a clear oil (7.64 g, $50 \%$ from methyl $\alpha$-D-mannopyranoside); $[\alpha]_{\mathrm{D}}+129.6^{\circ}\left(c 0.5, \mathrm{CHCl}_{3}\right) ;{ }^{1} \mathrm{H} \mathrm{NMR}\left(300 \mathrm{MHz}, \mathrm{CDCl}_{3}\right)$ : $\delta 5.67$ (apparent dt, 1H, $J=2.0$ and 9.5), $5.36(\mathrm{~d}, 1 \mathrm{H}, J=3.0), 5.28(\mathrm{dd}, 1 \mathrm{H}, J=3.0$ and 9.5), 5.19 (apparent t, 1H, $J=9.5$ ), 4.95 (apparent t, 1H, $J=2.0$ ), 3.18 (apparent t, 1H, $J=2.0$ ), 2.16, 2.15, 2.03 (each s, 9H, each $\left.\mathrm{CH}_{3}\right) ;{ }^{13} \mathrm{C}$ NMR $\left(125 \mathrm{MHz} \mathrm{CDCl}_{3}\right): \delta 169.9,169.7,169.6$ (each s, each $\left.\mathrm{C}=\mathrm{O}\right), 151.3$ (s, C-5), 99.4 (t, C-6), 87.5 (d, C-1), 69.0, 68.2, 67.2 (each d), 20.9, 20.9, 20.8 (each q, each $C \mathrm{H}_{3}$ ); IR $(\mathrm{KBr}): 2118\left(\mathrm{~N}_{3}\right) 1751(\mathrm{C}=\mathrm{O}), 1663(\mathrm{C}=\mathrm{C})$, 1432, 1373, $1210 \mathrm{~cm}^{-1}$. CI-HRMS: Found 331.1249, required $331.1254\left(\mathrm{M}+\mathrm{NH}_{4}\right)^{+}$.

\section{2,3,4-Tri- $O$-acetyl-5,6-anhydro-5- $C$-hydroxy- $\alpha$-D-mannopyranosyl azide and 2,3,4-tri- $O$-acetyl- 5,6-anhydro-5- $C$-hydroxy- $\beta$-L-gulopyranosyl azide 11 a}

To a cold $\left(0{ }^{\circ} \mathrm{C}\right)$ solution of $\mathbf{1 0 a}(0.5 \mathrm{~g}, 1.6 \mathrm{mmol})$ in acetonitrile $(10 \mathrm{~mL})$ was added aqueous sodium EDTA $(9 \mathrm{~mL}, 0.4 \mathrm{M})$, then 1,1,1 trifluoroacetone $(1.2 \mathrm{~mL}, 12.8 \mathrm{mmol})$ via a precooled syringe. To this was added sodium hydrogen carbonate $(0.8 \mathrm{~g}, 9.0 \mathrm{mmol})$ and oxone $(3.5 \mathrm{~g}, 6.5 \mathrm{mmol})$, portionwise over $1 \mathrm{~h}$. The resulting suspension was allowed to warm to room temp and after a further $0.5 \mathrm{~h}$ the reaction was poured onto ice $(25 \mathrm{~g})$. The resulting solution was extracted into $\mathrm{CH}_{2} \mathrm{Cl}_{2}(3 \times 50 \mathrm{~mL})$, washed several times with water and concentrated in vacuo to provide a mixture of epoxides 11 a (97\%), which was used in the next reaction without further purification; CI-HRMS: Found 347.1202, required $347.1203\left(\mathrm{M}+\mathrm{NH}_{4}\right)^{+} ;{ }^{1} \mathrm{H} \mathrm{NMR}$ (Isomer 1, $\left.300 \mathrm{MHz}, \mathrm{CDCl}_{3}\right): \delta 5.79(\mathrm{~d}, 1 \mathrm{H}, J=10.5), 5.53(\mathrm{dd}, 1 \mathrm{H}, J=$ 3.5 and 10.5), 5.39 (d, 1H, $J=2.0), 5.27(\mathrm{dd}, 1 \mathrm{H}, J=2.0$ and 10.5), $2.97(\mathrm{~d}, 1 \mathrm{H}, J=4.0), 2.65(\mathrm{~d}, 1 \mathrm{H}, J$ =4.0), 2.18, 2.07, 2.01 (each s, 9H); ${ }^{13} \mathrm{C} \mathrm{NMR}\left(125 \mathrm{MHz}, \mathrm{CDCl}_{3}\right.$ ): $\delta$ 170.2, 169.9, 169.6 (each s, each $\mathrm{C}=\mathrm{O}$ ), 87.7 (d, C-1), 80.8 (s, C-5), 69.5, 66.7, 64.1 (each d), 47.3 (t, C-6), 20.9, 20.8, 20.7 (each q, each $\mathrm{CH}_{3}$ ); ${ }^{1} \mathrm{H}$ NMR (Isomer 2, $\left.300 \mathrm{MHz}, \mathrm{CDCl}_{3}\right): \delta 5.39(\mathrm{~d}, 1 \mathrm{H}, J=6.5), 5.37(\mathrm{dd}, 1 \mathrm{H}, J=3.0$ and 6.5), $5.39(\mathrm{dd}, 1 \mathrm{H}, J=2.0$ and 6.5), $5.27(\mathrm{~d}, J=2.0), 2.90(\mathrm{~d}, 1 \mathrm{H}, J=4.5), 2.96(\mathrm{~d}, 1 \mathrm{H}, J=4.5), 2.14,2.12$, 2.11 (each s, 9H).

\section{2,3,6-Tri- $O$-acetyl-5- $C$-methoxy- $\beta$-L-gulopyranoside 13}

The mixture of epoxides $11 \mathrm{a}(0.16 \mathrm{~g}, 0.46 \mathrm{mmol})$ were dissolved in $(5 \mathrm{~mL})$ and heated at reflux overnight. The solvent was removed in vacuo and chromatography gave 13 (0.17 g, $98 \%$ \%) ${ }^{1} \mathrm{H}$ NMR $\left(300 \mathrm{MHz}, \mathrm{CDCl}_{3}\right): \delta 5.25$ (apparent t, $\left.1 \mathrm{H}, J=3.5\right), 5.19(\mathrm{dd}, 1 \mathrm{H}, J=9.0$ and 3.5), $5.05(\mathrm{~d}, 1 \mathrm{H}, J=9.0)$, $4.42(\mathrm{~d}, 1 \mathrm{H}, J=12.5), 2.97(\mathrm{~d}, 1 \mathrm{H}, J=12.5), 3.88($ apparent t, $1 \mathrm{H}, J=3.5), 3.79(\mathrm{~d}, 1 \mathrm{H}, J=3.5), 3.37(\mathrm{~s}$, 
3H) 2.12, 2.10, 2.09 (each s, 9H); ${ }^{13} \mathrm{C} \mathrm{NMR} \mathrm{(125} \mathrm{MHz,} \mathrm{CDCl}_{3}$ ): $\delta 171.5,170.7,169.7$ (each s, each $\mathrm{C}=\mathrm{O}$ ), 101.7 (s, C-5), 81.4 (d, C-1), 70.8, 66.9, 66.8 (each d), 59.9 (t, C-6), 49.0 (q, OCH ), 21.1, 21.0, 20.9 (each q, each $\left.\mathrm{CH}_{3}\right)$; CI-HRMS: Found 379.1468, required 379.1465 $\left(\mathrm{M}+\mathrm{NH}_{4}\right)^{+}$.

\section{5-C-Methoxy- $\beta$-L-gulopyranosyl azide 14}

Compound 13 (0.12 g, $0.32 \mathrm{mmol})$ was dissolved in $\mathrm{MeOH}(5 \mathrm{~mL})$ and elemental sodium (10 mg) was added. The solution was stirred for $4 \mathrm{~h}$ and then amberlite IR-120 (plus) ion exchange resin was added and stirring continued. After 10 min. the solution was filtered and concentrated to give $\mathbf{1 4}$ (quantitative) as a pale brown oil. Attempts to purify by chromatography led to substantial loss of 14 by decomposition and so the residue from the reaction was used without purification in the next step; $[\alpha]_{\mathrm{D}}$ $+83.6^{\circ}$ (c 0.75, MeOH); ${ }^{1} \mathrm{H}$ NMR $\left(300 \mathrm{MHz}, \mathrm{D}_{2} \mathrm{O}\right): \delta 5.02(\mathrm{~d}, 1 \mathrm{H}, J=9.0), 4.02$ (br s, $\left.1 \mathrm{H}\right), 3.91$ (br s, 1H), 3.76-3.64 (overlapping signals, 3H), 3.34 (s, 3H); ${ }^{13} \mathrm{C}$ NMR (125 MHz): $\delta 103.3$ (d, C-5), 84.0 (d, C-1), 71.6, 68.0, 67.2 (each d), 57.7 (t, C-6), 27.7 (q, $\left.\mathrm{OCH}_{3}\right)$; IR (KBr): $3414(\mathrm{OH}), 2123\left(\mathrm{~N}_{3}\right), 1247$, $1073 \mathrm{~cm}^{-1}$. CI-HRMS: Found 253.1145, required $253.1148\left(\mathrm{M}+\mathrm{NH}_{4}\right)^{+}$.

\section{1-Deoxymannojirimycin 2}

Compound 14 (0.40 g, $1.7 \mathrm{mmol})$ was dissolved in ethanol $(20 \mathrm{~mL})$ and shaken in a Parr Hydrogenator under an atmosphere of hydrogen (40 p.s.i) in the presence of $\mathrm{Pd}-\mathrm{C}(0.1 \mathrm{~g})$ for $12 \mathrm{~h}$. The suspension was filtered and the solvent was removed in vacuo to give the title compound $(0.23 \mathrm{~g})$. Some of the residue $(0.15 \mathrm{~g})$ was dissolved in $\mathrm{MeOH}(20 \mathrm{~mL})$ and $\mathrm{HCl}\left(1 \mathrm{M}\right.$ in $\left.\mathrm{Et}_{2} \mathrm{O}, 1.5 \mathrm{~mL}\right)$ was added. The solvents were evaporated and the salt was chromatographed (eluting with -chloroform-triethylamine, 100:100:1). This gave the free amine (64 mg, $23 \%$ ); treatment with $\mathrm{HCl}$ as above gave the hydrochloride salt. The NMR data of the salt agreed with those of an authentic sample purchased from Sigma; ${ }^{1} \mathrm{H}$ NMR $(300 \mathrm{MHz}$, $\mathrm{D}_{2} \mathrm{O}, \mathrm{HCl}$ salt): $\delta=4.09$ (apparent dt, $1 \mathrm{H}, J=2.7,2.7$ and 1.2), $3.82(\mathrm{dd}, 1 \mathrm{H}, J=12.0$ and 3.0), 3.69 (apparent t, 1H, J = 9.6), 3.64 (dd, $\mathrm{J}=5.2$ and 12.0), $3.53(\mathrm{dd}, 1 \mathrm{H}, \mathrm{J}=2.7$ and 9.6), $3.24(\mathrm{dd}, 1 \mathrm{H}, \mathrm{J}=2.7$ and 14.4), $3.06\left(\mathrm{dd}, 1 \mathrm{H}, J=1.2\right.$ and 14.4), $2.96(\mathrm{ddd}, 1 \mathrm{H}, J=5.2,3.3$ and 9.6$) ;{ }^{13} \mathrm{C} \mathrm{NMR}\left(75 \mathrm{MHz}, \mathrm{D}_{2} \mathrm{O}\right.$, free base) $\delta 48.2$ (C-1), 60.3 (C-6), 60.6 (C-5), 67.9, 68.6, 74.2 (C-2, C-3, C-4).

\section{Methyl 2,3,4-tri-O-acetyl-6-deoxy-6-iodo- $\alpha$-D-glucopyranoside 9b}

Methyl $\alpha$-D-glucopyranoside (9 g, $46.3 \mathrm{mmol}$ ) was treated as described above for methyl $\alpha$-Dmannopyranoside and gave the title compound ${ }^{1}$ which was used without purification in the next 
reaction; $+114.6^{\circ}$ (c 0.56, acetone); ${ }^{1} \mathrm{H}$ NMR (300 $\left.\mathrm{MHz}, \mathrm{CDCl}_{3}\right): \delta 5.46(\mathrm{dd}, 1 \mathrm{H}, J=9.4$ and 10.1$)$, $4.96(\mathrm{~d}, 1 \mathrm{H}, J=3.7), 4.88(\mathrm{dd}, 1 \mathrm{H}, J=3.7$ and 10.1), 4.87 (apparent t, 1H, $J=10.1), 3.79$ (ddd, 1H, $J=$ 9.4, 2.5 and 8.3), $3.48(\mathrm{~s}, 3 \mathrm{H}), 3.30(\mathrm{dd}, 1 \mathrm{H}, J=10.9$ and 2.5), $3.13(\mathrm{dd}, 1 \mathrm{H}, J=10.9$ and 8.3), 2.07, 2.05, 2.0 (each s, 9H); ${ }^{13} \mathrm{C}$ NMR $\left(125 \mathrm{MHz} \mathrm{CDCl}_{3}\right)$ : $\delta 199.5,170.0,169.9$ (each s, each $\left.\mathrm{C}=\mathrm{O}\right), 96.6(\mathrm{~d}$, C-1), 72.4, 70.8, 69.0, 68.6 (each d), 55.7 (q, $\mathrm{OCH}_{3}$ ), 20.6, 20.6, 20.5 (each q, each $\mathrm{CH}_{3}$ ), 3.79 (t, C-6); IR (KBr): $1748(\mathrm{C}=\mathrm{O}), 1376,1245,1135,1042,972 \mathrm{~cm}^{-1}$. Anal. Calcd. for $\mathrm{C}_{13} \mathrm{H}_{19} \mathrm{O}_{8} \mathrm{I}: \mathrm{C}, 36.28 ; \mathrm{H}$, 4.42; I, 29.54. Found: C, 36.24; H, 4.48; I, 29.62. CI-HRMS: Found 448.0470, required 448.0468 $\left(\mathrm{M}+\mathrm{NH}_{4}\right)^{+}$.

\section{1,2,3,4-Tetra- $O$-acetyl-6-deoxy-6-iodo- $\alpha$-D-glucopyranose}

All of the unpurified iodide 9a obtained as described above was dissolved in acetic anhydride and sulphuric acid $(50: 1,250 \mathrm{~mL})$ and the mixture was stirred for $12 \mathrm{~h}$ at $\mathrm{rt}$. The solution was then concentrated in vacuo and residue was separated between EtOAc and water. The organic layer was washed with aq. $\mathrm{NaHCO}_{3}(5 \times 500 \mathrm{~mL})$, water $(2 \times 500 \mathrm{~mL})$ and dried and the title compound was obtained as a pale yellow oil after the removal of solvent. A small portion was further purified by chromatography (EtOAc: petroleum ether gradient commencing with 1:4) so as to obtain an analytical sample of the title compound; $[\alpha]_{\mathrm{D}}+86.5^{\circ}\left(c 0.2, \mathrm{CHCl}_{3}\right) ;{ }^{1} \mathrm{H} \mathrm{NMR}\left(300 \mathrm{MHz}, \mathrm{CDCl}_{3}\right): \delta 6.34(\mathrm{~d}, 1 \mathrm{H}, J$ = 3.7), 5.47 (apparent t, 1H, $J=9.8), 5.08(\mathrm{dd}, 1 \mathrm{H}, J=3.7$ and 9.8), 4.98 (apparent t, 1H, $J=9.8), 3.83$ (ddd, $1 \mathrm{H}, J=2.9$ and 6.3), $3.31(\mathrm{dd}, 1 \mathrm{H}, J=11.2$ and 2.9), $3.14(\mathrm{dd}, 1 \mathrm{H}, J=11.2$ and 6.3), 2.18, 2.07, 2.02, 2.01 (each s, each $\mathrm{CH}_{3}$ ); ${ }^{13} \mathrm{C}$ NMR (125 MHz, $\mathrm{CDCl}_{3}$ ): $\delta 170.0,169.8,169.5,167.1$ (each s, each $\mathrm{C}=\mathrm{O}$ ), 90.8 (d, C-1), 72.1, 69.9, 68.6, 68.5 (each d), 21.0, 20.9, 20.8, 20.6 (each q, each $\mathrm{CH}_{3}$ ), 3.7 (t, C6); IR (KBr): 1748, 1371, 1220, 1151, $1058 \mathrm{~cm}^{-1}$. CI-HRMS: Found 476.0416, required 476.0418 $\left(\mathrm{M}+\mathrm{NH}_{4}\right)^{+}$. Anal. Calcd. for $\mathrm{C}_{14} \mathrm{H}_{19} \mathrm{O}_{9} \mathrm{I}$ : C, 36.70; H, 4.18; I, 27.7. Found: C, 36.79; H, 4.01; I, 27.51.

\section{2,3,4-Tri- $O$-acetyl-6-deoxy-6-iodo- $\beta$-D-glucopyranosyl azide}

To a solution of 1,2,3,4-tetra- $O$-acetyl-6-deoxy-6-iodo- $\alpha$-D-glucopyranose, used as obtained in the above reaction without purification, in $\mathrm{CH}_{2} \mathrm{Cl}_{2}(250 \mathrm{~mL})$ under an inert atmosphere was added first $\mathrm{SnCl}_{4}(3.0 \mathrm{~mL}, 25 \mathrm{mmol})$, then azidotrimethylsilane $(10.0 \mathrm{~mL}, 76 \mathrm{mmol})$. The resulting solution was stirred at room temp for $10 \mathrm{~min}$, then diluted with $\mathrm{CH}_{2} \mathrm{Cl}_{2}(300 \mathrm{~mL})$, washed with aq. $\mathrm{NaHCO}_{3}(4 \times 500$ $\mathrm{mL})$, water $(2 \times 500 \mathrm{~mL})$ and dried over magnesium sulphate. The solvent was removed in vacuo to give the title compound as a pale oil; $[\alpha]_{\mathrm{D}}+86.5^{\circ}\left(c 0.2, \mathrm{CHCl}_{3}\right) ;{ }^{1} \mathrm{H} \mathrm{NMR}\left(300 \mathrm{MHz}, \mathrm{CDCl}_{3}\right): \delta 5.23$ 
(apparent t, 1H, $J=9.4$ ), 4.95 (2 x overlapping apparent t, 2H, $J=9.4), 4.70$ (d, 1H, $J=9.4), 3.57$ (ddd, $1 \mathrm{H}, J=2.6,7.2$ and 9.4), $3.34(\mathrm{dd}, 1 \mathrm{H}, J=11.1$ and 2.6), $3.17(\mathrm{dd}, 1 \mathrm{H}, J=11.1$ and 7.2), 2.07, 2.07, 2.06, 2.06 (each s, 12H); ${ }^{13} \mathrm{C}$ NMR $\left(125 \mathrm{MHz} \mathrm{CDCl}_{3}\right): \delta 170.0,169.9,169.8$, (each s, each C=O), 87.6 (d, C-1), 72.2, 69.7, 69.6, 68.2 (each d), 20.0, 19.0, 18.7, (each q, each $\mathrm{CH}_{3}$ ), 3.7 (t, C-6); IR (thin film): $2120\left(\mathrm{~N}_{3}\right), 1742,1376,1321,1126,1090,820 \mathrm{~cm}^{-1}$. CI-HRMS: Found 459.0373, required 459.0377 $\left(\mathrm{M}+\mathrm{NH}_{4}\right)^{+}$.

\section{2,3,4-Tri-O-acetyl-6-deoxy- $\beta$-D-xylo-hex-5-enopyranosyl azide 10b}

A solution of 2,3,4-tri-O-acetyl-6-deoxy-6-iodo- $\beta$-D-glucopyranosyl azide and DBU (14.4 mL, 96.4 mmol) in toluene $(150 \mathrm{~mL})$ was stirred and heated at reflux for $1.5 \mathrm{~h}$, after which time the UV active iodide starting material had been consumed. The solution was washed with water, the solvent was removed and the residue chromatographed (EtOAc: petroleum ether gradient, commencing with 1:6, as eluant) to give the title compound ${ }^{2}$ as a clear oil (7.53 g, 52\% from methyl $\alpha$-D-glucopyranoside); $[\alpha]_{\mathrm{D}}$ $40^{\circ}$ (c 0.08, acetone); ${ }^{1} \mathrm{H}$ NMR (300 MHz, $\mathrm{CDCl}_{3}$ ): $\delta 5.52$ (apparent dt, $1 \mathrm{H}, J=1.6$ and 7.5), 5.16 (apparent t, 1H, $J=7.5$ ), 4.98 (apparent t, 1H, $J=7.5$ ), 4.93-5.04 (overlapping d $(J=7.5$ ) and apparent t $(J=1.6), 2 \mathrm{H}), 4.65$ (apparent t, $1 \mathrm{H}, J=1.6), 2.12,2.09,2.05$ (each s, each $\left.\mathrm{CH}_{3}\right) ;{ }^{13} \mathrm{C} \mathrm{NMR}(125 \mathrm{MHz}$, $\left.\mathrm{CDCl}_{3}\right): \delta 169.9,169.3,169.2$ (each s, each $\mathrm{C}=\mathrm{O}$ ), $150.7(\mathrm{~s}, \mathrm{C}-5), 98.3(\mathrm{t}, \mathrm{C}-6), 88.2(\mathrm{~d}, \mathrm{C}-1), 71.6$, 70.9, 68.6 (each d), 20.9, 20.8, 20.77 (each q, each $\left.\mathrm{CH}_{3}\right)$; IR (KBr): $2105\left(\mathrm{~N}_{3}\right), 1749(\mathrm{C}=\mathrm{O}), 1635,1432$, 1375, $1048 \mathrm{~cm}^{-1}$. Anal. Calcd. for $\mathrm{C}_{12} \mathrm{H}_{15} \mathrm{O}_{7} \mathrm{~N}_{3}: \mathrm{C}, 46.12 ; \mathrm{H}, 4.81 ; \mathrm{N}, 13.25$. Found: $\mathrm{C}, 46.01 ; \mathrm{H}, 4.83 ; \mathrm{N}$, 13.41 .

\section{2,3,4-Tri- $O$-acetyl-5,6-anhydro-5- $C$-hydroxy- $\beta$-D-glucopyranosyl azide and 2,3,4-tri- $O$-acetyl-5,6- anhydro-5- $C$-hydroxy- $\alpha$-L-idopyranosyl azide $11 b$}

To a cold $\left(0{ }^{\circ} \mathrm{C}\right)$ solution of $\mathbf{1 0 b}(0.5 \mathrm{~g}, 1.6 \mathrm{mmol})$ in acetonitrile $(10 \mathrm{~mL})$ was added aqueous sodium EDTA $(9 \mathrm{~mL}, 0.4 \mathrm{M})$, then 1,1,1 trifluoroacetone $(1.2 \mathrm{~mL}, 12.8 \mathrm{mmol})$ via a precooled syringe. To this was added sodium hydrogen carbonate $(0.8 \mathrm{~g}, 9.0 \mathrm{mmol})$ and oxone $(3.5 \mathrm{~g}, 6.5 \mathrm{mmol})$, portionwise over $1 \mathrm{~h}$. The resultant suspension was allowed to warm to room temp and after a further $0.5 \mathrm{~h}$, was poured onto ice $(25 \mathrm{~g})$. The products were extracted into $\mathrm{CH}_{2} \mathrm{Cl}_{2}(3 \times 50 \mathrm{~mL})$, washed several times with water and the solvent then removed in vacuo (95\%) to give the title compounds as a 1:1.7 mixture. Anal. Calcd. for $\mathrm{C}_{12} \mathrm{H}_{15} \mathrm{O}_{8} \mathrm{~N}_{3}$ (mixture): C, 43.77; H, 4.59; N, 12.76. Found: C, 43.86; H, 4.75; N, 12.79 . 
NMR data for isomer 1: ${ }^{1} \mathrm{H}$ NMR $\left(300 \mathrm{MHz}, \mathrm{CDCl}_{3}\right): \delta 5.53(\mathrm{~d}, 1 \mathrm{H}, J$ 9.5), 5.43 (apparent $\mathrm{t}, 1 \mathrm{H}, J=$ 9.5), 5.1 (apparent t, 1H, $J=9.5), 4.97(\mathrm{~d}, 1 \mathrm{H}, J=9.5), 3.15(\mathrm{~d}, 1 \mathrm{H}, J=4.0), 2.78(\mathrm{~d}, 1 \mathrm{H}, J=4.0), 2.09$, 2.04, 2.02 (each s, 9H); ${ }^{13} \mathrm{C}$ NMR (125 MHz, $\mathrm{CDCl}_{3}$ ), $\delta 169.8,169.7,169.4$ (each s, each C=O), 86.1 (d, C-1), 80.7, (s, C-5), 70.8, 70.7, 66.1 (each d), 49.8 (t, C-6), 20.7, 20.6 (each q, each $\mathrm{CH}_{3}$ ).

NMR data for isomer 2: ${ }^{1} \mathrm{H}-\mathrm{NMR}\left(300 \mathrm{MHz} \mathrm{CDCl}_{3}\right): \delta 5.34(\mathrm{~d}, 1 \mathrm{H}, J=7.5), 5.28$ (apparent t $, 1 \mathrm{H}, J=$ 7.5), 5.09 (apparent t, 1H, $J=7.5), 4.95$ (d, 1H, $J=7.5), 2.97$ (d, 1H, $J=4.9), 2.95$ (d, 1H, $J=4.9), 2.11$, 2.06, 2.05 (each s, 9H); ${ }^{13} \mathrm{C}$ NMR (125 MHz, $\mathrm{CDCl}_{3}$ ), $\delta 170.0,169.3,169.0$ (each s, each $\left.\mathrm{C}=\mathrm{O}\right), 87.1$ (d, C-1), 80.8, (s, C-5), 70.9, 70.3, 67.5 (each d), 49.0 (t, C-6), 20.8 and 20.5 (each q, each $\mathrm{CH}_{3}$ ).

\section{Acyclic compound 19}

Epoxides 11b (0.75 g, $2.0 \mathrm{mmol})$ were dissolved in $(23 \mathrm{~mL})$ and the mixture was heated at reflux overnight. The solution was allowed to cool and pyridine $(7.0 \mathrm{~mL})$ and acetic anhydride $(7.0 \mathrm{~mL})$ were added. The solution was stirred for $30 \mathrm{~min}$ after which time xylene was added and the volatile substances were removed in vacuo. The residue was purified by chromatography (EtOAc: petroleum ether gradient, commencing with $1: 3$, as eluant) which gave 19 as a white solid $(0.45 \mathrm{~g}, 58 \%)$; $[\alpha]_{\mathrm{D}}$ $+42.7^{\circ}\left(c 0.61, \mathrm{CHCl}_{3}\right) ;{ }^{1} \mathrm{H}$ NMR $\left(300 \mathrm{MHz}, \mathrm{CDCl}_{3}\right.$ ): $\delta 5.80$ (overlapping dd and d, $2 \mathrm{H}, J=2.8$ and 6.5 ), $5.61(\mathrm{~d}, 1 \mathrm{H}, J=3.4), 5.34(\mathrm{dd}, 1 \mathrm{H}, J=6.5$ and 2.8), $4.12(2 \mathrm{x} \mathrm{d}, 2 \mathrm{H}, J=17.0), 3.46,3.42$ (each s, 6H), 2.18, 2.13, 2.08, 2.04 (each s, 12H); ${ }^{13} \mathrm{C} \mathrm{NMR}\left(125 \mathrm{MHz}, \mathrm{CDCl}_{3}\right.$ ): $\delta$ 201.6, 170.4, 170.2, 170.0, 169.8 (each s, each $\mathrm{C}=\mathrm{O}$ ), 95.5 (d), 76.5 (t), 74.3, 70.8, 68.3 (each d), 59.7, 57.7 (each q, each $\mathrm{OCH}_{3}$ ), 21.0, 20.7, 20.7, 20.5 (each q, each $\mathrm{CH}_{3}$ ); IR (KBr): 2941, $2124\left(\mathrm{~N}_{3}\right), 1750(\mathrm{C}=\mathrm{O}), 1432,1376,1213$; CIHRMS: Found 410.1665, required $410.1662\left(\mathrm{M}+\mathrm{NH}_{4}\right)^{+}$. Anal. Calcd. for $\mathrm{C}_{16} \mathrm{H}_{24} \mathrm{O}_{11}$ : C, 48.98; $\mathrm{H}, 6.17$; N. Found: C, 48.97; H, 6.13.

\section{1,2:3,4-Di- $O$-isopropylidene-6-deoxy-6- $\alpha$-iodo-D-galactopyranoside}

To 1,2:3,4-di- $O$-propylidene- $\alpha$-D-galactopyranoside $(25 \mathrm{~g}, 96.1 \mathrm{mmol})$ in toluene $(400 \mathrm{~mL})$ at $80{ }^{\circ} \mathrm{C}$, triphenylphosphine $(15.4 \mathrm{~g}, 115.3 \mathrm{mmol})$, imidazole $(31.4 \mathrm{~g}, 461.2 \mathrm{mmol})$ and iodine $(48.8 \mathrm{~g}, 192.2$ mmol) were added successively in small portions. The mixture was allowed to stir for $10 \mathrm{~h}$, cooled to room temp and washed with 10\% aqueous sodium thiosulphate $(2 \times 400 \mathrm{~mL})$, water $(2 \times 400 \mathrm{~mL})$, dried $\left(\mathrm{Na}_{2} \mathrm{SO}_{4}\right)$, filtered, and the solvent removed in vacuo. Chromatography (6:1 petroleum ether-EtOAc) gave the title compound as a white solid (33.4 g, $94 \%)$; $\mathrm{R}_{\mathrm{F}} 0.76$ (3:1 petroleum ether-EtOAc); m.p. 64$66{ }^{\circ} \mathrm{C}[\alpha]_{\mathrm{D}}-44.2^{\circ}\left(c 0.5 \mathrm{CHCl}_{3}\right) ;{ }^{1} \mathrm{H} \mathrm{NMR}\left(\mathrm{CDCl}_{3}, 300 \mathrm{MHz}\right) ; \delta 5.55(\mathrm{~d}, 1 \mathrm{H}, J 5.0), 4.63(\mathrm{dd}, 1 \mathrm{H}, J=$ 
7.9 and 2.5), $4.42(\mathrm{dd}, 1 \mathrm{H}, J=7.9$ and 1.9), $4.31(\mathrm{dd}, 1 \mathrm{H}, J=5.0$ and 2.5), 3.96 (apparent dt, $1 \mathrm{H}, J=7.0$ and 1.9), $3.33(\mathrm{dd}, 1 \mathrm{H}, J=10.0$ and 7.0), $3.22(\mathrm{dd}, 1 \mathrm{H}, J=10.0$ and 7.0), 1.56, 1.46, 1.37, 1.35 (each s, $12 \mathrm{H}) ;{ }^{13} \mathrm{C} \mathrm{NMR}\left(\mathrm{CDCl}_{3}, 125 \mathrm{MHz}\right) ; \delta 109.7,109.0$ (each s, 2 x $\left.C\left(\mathrm{CH}_{3}\right)_{2}\right), 96.9$ (d, C-1), 71.7, 71.3, 70.8, 69.2 (each d, C-2-5), 26.2, 26.1, 25.0, 24.6 (each q, 4 x C $\left.\left(\mathrm{CH}_{3}\right)_{2}\right), 2.4$ (t, C-6); IR (KBr) v 2989, 1383, $1066 \mathrm{~cm}^{-1}$. Anal. Calcd. for $\mathrm{C}_{12} \mathrm{H}_{19} \mathrm{IO}_{5}$ requires $\mathrm{C}, 38.93$; H, 5.17; I, 34.28; Found C, 38.85; H, 5.06; I, 34.48; HRMS-CI: Found 388.0621, required $388.0622\left[\mathrm{M}+\mathrm{NH}_{4}\right]^{+}$.

\section{1,2,3,4-Tetra- $O$-acetyl-6-deoxy-6-iodo-D-galactopyranose}

To 1,2:3,4-Di-O-isopropylidene-6-deoxy-6-iodo-D-galactopyranoside (15 g, $40.5 \mathrm{mmol})$ stirring in acetic acid $(300 \mathrm{~mL})$ at $0{ }^{\circ} \mathrm{C}$, acetic anhydride $(75 \mathrm{~mL})$ and $\mathrm{H}_{2} \mathrm{SO}_{4}(40 \mathrm{~mL})$ was added slowly over 20 min. The solution was allowed to warm to $35^{\circ} \mathrm{C}$ and was stirred at this temperature for a further 2 days. The mixture was poured onto ice water $(300 \mathrm{~mL})$ and extracted with $\mathrm{CH}_{2} \mathrm{Cl}_{2}(2 \times 300 \mathrm{~mL})$. The combined organic layers were washed with water $(2 \times 300 \mathrm{~mL})$, dried $\left(\mathrm{Na}_{2} \mathrm{SO}_{4}\right)$, filtered and the solvent was removed in vacuo to give the title compound $(\alpha: \beta, 5: 1)$ as a yellow oil $(11.6 \mathrm{~g}, 62 \%) ; \mathrm{R}_{\mathrm{F}} 0.40(2: 1$ petroleum ether-EtOAc); HRMS-CI: Found 476.0414 required $476.0418\left[\mathrm{M}+\mathrm{NH}_{4}\right]^{+}$.

\section{2,3,4-Tri- $O$-acetyl-6-deoxy-6-iodo- $\beta$-D-galactopyranosyl azide}

To 1,2,3,4-tetra- $O$-acetyl-6-deoxy-6-iodo-D-galactopyranose $(6.33 \mathrm{~g}, 13.8 \mathrm{mmol})$ in $\operatorname{dry~} \mathrm{CH}_{2} \mathrm{Cl}_{2}(90 \mathrm{~mL})$ under a $\mathrm{N}_{2}$ atmosphere at $0{ }^{\circ} \mathrm{C}, \mathrm{SnCl}_{4}(0.81 \mathrm{~mL}, 6.9 \mathrm{mmol})$ was added dropwise and mixture was stirred for $20 \mathrm{~min}$. Azidotrimethylsilane $(3.66 \mathrm{~mL}, 27.6 \mathrm{mmol})$ was then added dropwise and the resulting solution was allowed warm to and stirred at room temp for a further $16 \mathrm{~h}$. The mixture was diluted with $\mathrm{CH}_{2} \mathrm{Cl}_{2}(50 \mathrm{~mL})$ and washed successively with aq. $\mathrm{NaHCO}_{3}(150 \mathrm{~mL})$ and brine $(150 \mathrm{~mL})$. The organic phase was dried $\left(\mathrm{Na}_{2} \mathrm{SO}_{4}\right)$, filtered and the solvent removed in vacuo. Chromatography (4:1 petroleum ether-EtOAc) gave the title compound ${ }^{2}$ as a white solid $(6.08 \mathrm{~g}, 91 \%)$; $\mathrm{R}_{\mathrm{F}} 0.55$ (1:1 petroleum etherEtOAc); m.p. $70-72{ }^{\circ} \mathrm{C} ;[\alpha]_{\mathrm{D}}+39.6^{\circ}\left(c 0.5, \mathrm{CHCl}_{3}\right) ;{ }^{1} \mathrm{H} \mathrm{NMR}\left(\mathrm{CDCl}_{3}, 300 \mathrm{MHz}\right) ; \delta 5.61\left(\mathrm{dd}, 1 \mathrm{H}, J_{4,3}\right.$ 3.2, 1.0, H-4), $5.13\left(\mathrm{dd}, 1 \mathrm{H}, J_{2,3} 10.4, J_{2,1} 8.5, \mathrm{H}-2\right), 5.04$ (dd, 1H, $J_{3,2} 10.4, J_{3,4} 3.2, \mathrm{H}-3$ ), 4.64 (d, $1 \mathrm{H}$, $J_{1,2} 8.5, \mathrm{H}-1$ ), 4.0 (apparent t, $J_{5,6 \mathrm{a}} 7.0, J_{5,6 \mathrm{~b}} 7.0,1 \mathrm{H}, \mathrm{H}-5$ ), 3.25 (dd, 1H, $\left.J_{6 \mathrm{~b}, 6 \mathrm{a}} 10.5, J_{6 \mathrm{~b}, 5} 7.0, \mathrm{H}-6 \mathrm{~b}\right), 3.16$ $\left(\mathrm{dd}, 1 \mathrm{H}, J_{6 \mathrm{a}, 6 \mathrm{~b}} 10.5, J_{6 \mathrm{a}, 5} 7.0, \mathrm{H}-6 \mathrm{a}\right), 2.19,2.09,2.05$ (each s, 9H, each $\left.\mathrm{CH}_{3}\right)$; ${ }^{13} \mathrm{C} \mathrm{NMR}\left(\mathrm{CDCl}_{3}\right.$, $125 \mathrm{MHz}$ ); $\delta 170.1,170.1,169.5$ (each s, each $\mathrm{C}=\mathrm{O}$ ), 88.3 (d, C-1), 76.0, 70.9, 68.1, 67.9 (each d, C-

\footnotetext{
${ }^{2}$ Györgydeák, Z.; Szilagyi, L. Liebigs Ann. Chem. 1987, 235.
} 
2-5), 20.8, 20.7, 20.6 (each q, each $\left.\mathrm{CH}_{3}\right),-1.1$ (t, C-6); IR (KBr) v 2946, $2119\left(\mathrm{~N}_{3}(, 1753(\mathrm{C}=\mathrm{O}), 1371\right.$, $1221 \mathrm{~cm}^{-1}$. Anal. Calcd. for $\mathrm{C}_{12} \mathrm{H}_{16} \mathrm{IN}_{3} \mathrm{O}_{5}$ requires $\mathrm{C}, 32.67$; H, 3.66; I, 28.77; N, 9.52; Found C, 32.63; H, 3.52; I, 28.60; N, 9.34. HRMS-CI: Found 459.0377 required $459.0377\left[\mathrm{M}+\mathrm{NH}_{4}\right]^{+}$.

\section{2,3,4-Tri- $O$-acetyl-6-deoxy- $\beta$-D-arabino-hex-5-enopyranosyl azide 20}

To 2,3,4-tri- $O$-acetyl-6-deoxy-6-iodo- $\beta$-D-galactopyranosyl azide $(5.30 \mathrm{~g}, 12.0 \mathrm{mmol})$ in dry $\mathrm{CH}_{2} \mathrm{Cl}_{2}(80$ $\mathrm{mL})$ under a $\mathrm{N}_{2}$ atmosphere at $35^{\circ} \mathrm{C}$, DBU $(9.05 \mathrm{~mL}, 60.0 \mathrm{mmol})$ was added. The solution was stirred for 2 days, cooled and was directly added to a column of silica gel and chromatographed (6:1 petroleum ether-EtOAc) to give $\mathbf{2 0}^{2}$ as a white solid (2.96 g, $78 \%$ ); $\mathrm{R}_{\mathrm{F}} 0.55$ (1:1 petroleum ether-EtOAc); m.p. 71$72{ }^{\circ} \mathrm{C} ;[\alpha]_{\mathrm{D}}-52.0^{\circ}\left(c 0.5 \mathrm{CHCl}_{3}\right) ;{ }^{1} \mathrm{H} \mathrm{NMR}\left(300 \mathrm{MHz}, \mathrm{CDCl}_{3}\right) ; \delta 5.72\left(\mathrm{~d}, 1 \mathrm{H}, J_{1,2} 3.5, \mathrm{H}-1\right), 5.25(\mathrm{dd}$, $\left.1 \mathrm{H}, J_{3,2} 8.8, J_{3,4} 6.7, \mathrm{H}-3\right), 5.11\left(\mathrm{dd}, 1 \mathrm{H} J_{2,3} 8.8, J_{2,1} 3.5, \mathrm{H}-2\right), 5.01$ (d, 1H, J6,6a $\left.1.6, \mathrm{H}-6\right), 4.85$ (d, $1 \mathrm{H}$, $J_{4,3}$ 6.7, H-4), 4.84 (d, 1H, $\left.J_{6 \mathrm{a}, 6} 1.6, \mathrm{H}-6 \mathrm{a}\right), 2.16,2.14,2.07$ (each s, 9H, each $\left.\mathrm{CH}_{3}\right) ;{ }^{13} \mathrm{C} \mathrm{NMR}(125 \mathrm{MHz}$, $\left.\mathrm{CDCl}_{3}\right) ; \delta 169.9,169.8,169.4$ (each s, each $\left.C=\mathrm{O}\right), 150.3(\mathrm{~s}, \mathrm{C}-5), 102.8(\mathrm{t}, \mathrm{C}-6), 88.6(\mathrm{~d}, \mathrm{C}-1), 69.3$, 68.2, 67.8 (each d, C-2-C-4), 20.9, 20.7, 20.6 (each q, each C=O); IR (KBr) v 2938, $2117\left(\mathrm{~N}_{3}\right), 1752$ $(\mathrm{C}=\mathrm{O}), 1368,1240,1071 \mathrm{~cm}^{-1}$. Anal. Calcd. for $\mathrm{C}_{12} \mathrm{H}_{15} \mathrm{~N}_{3} \mathrm{O}_{7}$ required: $\mathrm{C}, 46.01$; $\mathrm{H}, 4.83, \mathrm{~N}, 13.41$; Found C, 45.85; H, 4.75; N, 13.36; HRMS-CI: Found 331.1254, required 331.1257 [M+NH$]^{+}$.

\section{5-C-Methoxy- $\beta$-D-glucopyranosyl azide and 5- $C$-methoxy- $\alpha$-L-idopyranosyl azide}

These two isomers were obtained by deacetylation of 12. Analytical data for first isomer: $[\alpha]_{\mathrm{D}}-59.4^{\mathrm{o}}(c$ 0.5, MeOH); ${ }^{1} \mathrm{H}$ NMR (300 MHz, $\left.\mathrm{D}_{2} \mathrm{O}\right): \delta 4.92(\mathrm{~d}, 1 \mathrm{H}, \mathrm{J}=9.0), 3.91$ and $3.78($ each $\mathrm{d}$, each $1 \mathrm{H}, \mathrm{J}=12.9$ and 12.9), $3.85(\mathrm{~d}, 1 \mathrm{H}, \mathrm{J}=9.0), 3.70$ (apparent t, 1H, $\mathrm{J}=9.0), 3.46$ (apparent t, 1H, J=9.0), $3.26(\mathrm{~s}, 3 \mathrm{H})$; ${ }^{13} \mathrm{C}$ NMR (125 MHz): $\delta 101.6$ (s, C-5), 86.9 (d, C-1), 72.9, 72.8, 70.5 (each d), 58.9 (t, C-6), 27.7 (q, $\left.\mathrm{OCH}_{3}\right)$. Analytical data for second isomer: $[\alpha]_{\mathrm{D}}-93.3^{\circ}(c 0.14, \mathrm{MeOH}) ;{ }^{1} \mathrm{H}$ NMR $\left(300 \mathrm{MHz}, \mathrm{CD}{ }_{3} \mathrm{OD}\right)$ : $\delta 4.59$ (d, 1H, J 9.0), 3.75 and 3.72 (each d, each 1H, J = 11.7 and 11.7), 3.62 (apparent d, 2H, J 4.7), $3.36\left(\mathrm{~s}, 3 \mathrm{H}, \mathrm{OCH}_{3}\right), 3.16\left(\mathrm{dd}, 1 \mathrm{H}, \mathrm{J} 9.0\right.$ and 4.7, H-2); ${ }^{13} \mathrm{C} \mathrm{NMR}\left(125 \mathrm{MHz}, \mathrm{CD}_{3} \mathrm{OD}\right): \delta 100.5$ (s, C-5), 86.3 (d, C-1), 73.4, 72.7, 71.5 (each d), 50.8 (t, C-6), $47.8\left(\mathrm{q}, \mathrm{OCH}_{3}\right)$.

\section{NMR data for 1-deoxynojirimycin 1 (salt)}

${ }^{1} \mathrm{H}$ NMR (300 MHz, $\left.\mathrm{D}_{2} \mathrm{O}\right): \delta 3.78(\mathrm{dd}, 1 \mathrm{H}, \mathrm{J}=3.0$ and 12.0), $3.59(\mathrm{dd}, 1 \mathrm{H}, \mathrm{J}=6.0$ and 12.0), 3.45 (ddd, $1 \mathrm{H}, \mathrm{J}=5.1,10.9$ and 9.5), 3.28 (apparent $\mathrm{t}, 1 \mathrm{H}, \mathrm{J}=9.5), 3.21($ apparent $\mathrm{t}, 1 \mathrm{H}, \mathrm{J}=9.5), 3.10(\mathrm{dd}, 1 \mathrm{H}, \mathrm{J}=$ 
5.1 and 12.4), $2.55\left(\mathrm{ddd}, 1 \mathrm{H}, \mathrm{J}=9.5,6.0\right.$ and 3.0), $2.45(\mathrm{dd}, 1 \mathrm{H}, \mathrm{J}=10.9$ and 12.4$) ;{ }^{13} \mathrm{C} \mathrm{NMR}(75 \mathrm{MHz}$, $\left.\mathrm{D}_{2} \mathrm{O}\right) \delta 49.2,61.3,62.8,70.3,71.4,79.3$.

\section{NMR data for 1-deoxygalactostatin 3}

${ }^{1} \mathrm{H}$ NMR ( $\left.\mathrm{D}_{2} \mathrm{O}, 300 \mathrm{MHz}\right) ; \delta 4.10$ (apparent d, $\left.1 \mathrm{H}, J=3.0\right), 3.99$ (ddd, $\left.1 \mathrm{H}, J=11.5,10.0,5.0\right), 3.79$ (dd, $1 \mathrm{H}, \mathrm{J}=11.5,7.0), 3.74(\mathrm{dd}, 1 \mathrm{H}, \mathrm{J}=11.5,10.0), 3.56(\mathrm{dd}, 1 \mathrm{H}, \mathrm{J}=1.5$ and 7.0$) 3.41(\mathrm{dd}, 1 \mathrm{H}, \mathrm{J}=10.0$ and 3.0), $3.30(\mathrm{dd}, 1 \mathrm{H}, \mathrm{J}=12.5$ and 5.0), 2.79 (apparent t, 1H, $J=11.5$ ).

\section{NMR data for 1,6-dideoxygalactostatin 4}

$[\alpha]_{\mathrm{D}}+34^{\mathrm{o}}(c \mathrm{0.1}, \mathrm{MeOH}) ;{ }^{1} \mathrm{H}$ NMR $\left(\mathrm{D}_{2} \mathrm{O}, 300 \mathrm{MHz}\right) ; \delta 3.89-4.00(\mathrm{~m}, 2 \mathrm{H}), 3.57(\mathrm{dd}, 1 \mathrm{H}, J$ 9.9, 3.1), $3.37(\mathrm{~m}, 2 \mathrm{H}), 2.77(\mathrm{t}, 1 \mathrm{H}, J 11.7), 1.27$ (d, 3H, J 6.7). 
NMR Spectra

${ }^{1} \mathrm{H}-\mathrm{NMR}\left(\mathrm{CDCl}_{3}\right)$ of 9a

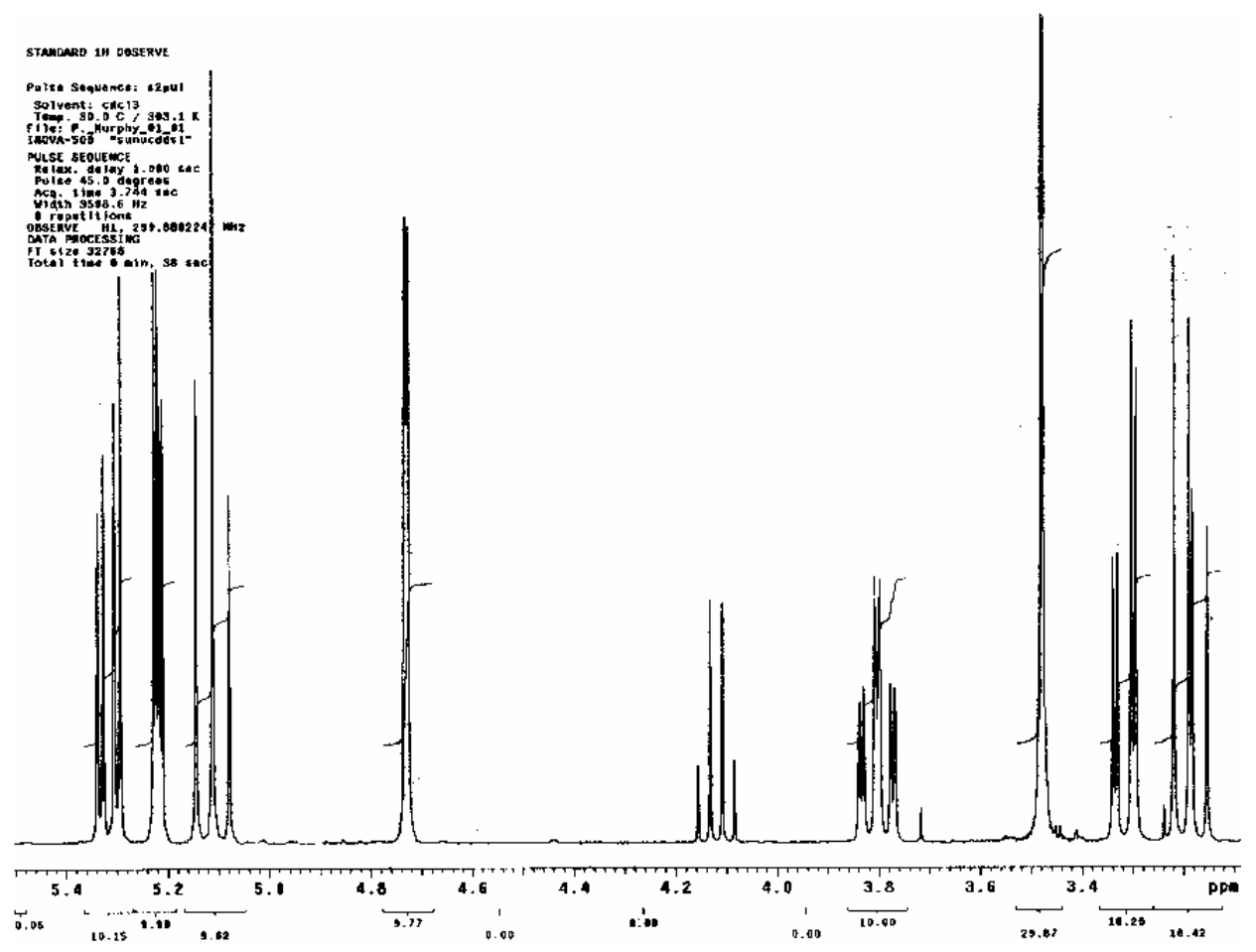

\section{${ }^{13} \mathrm{C}-\mathrm{NMR}\left(\mathrm{CDCl}_{3}\right)$ of 9a}

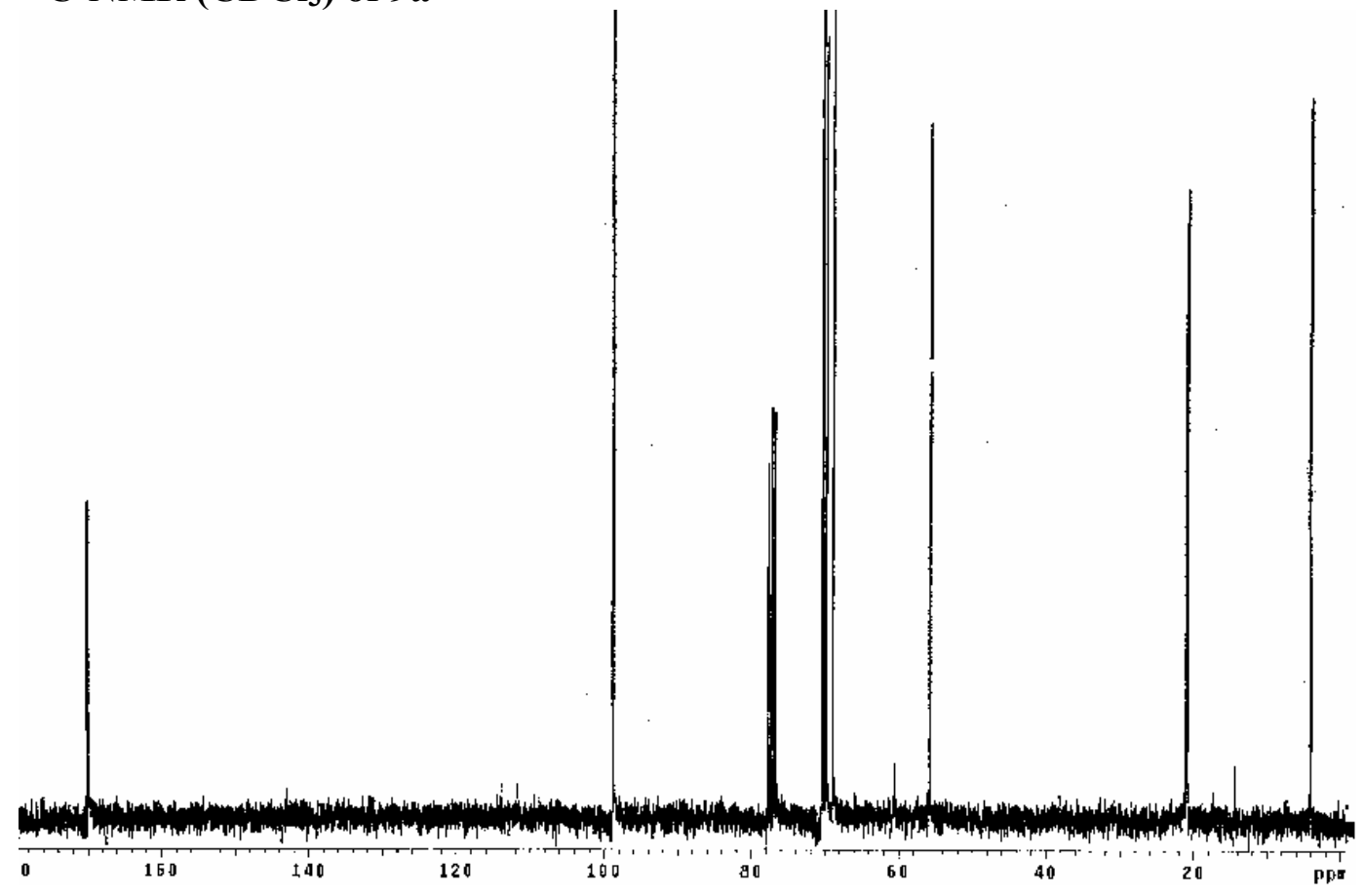



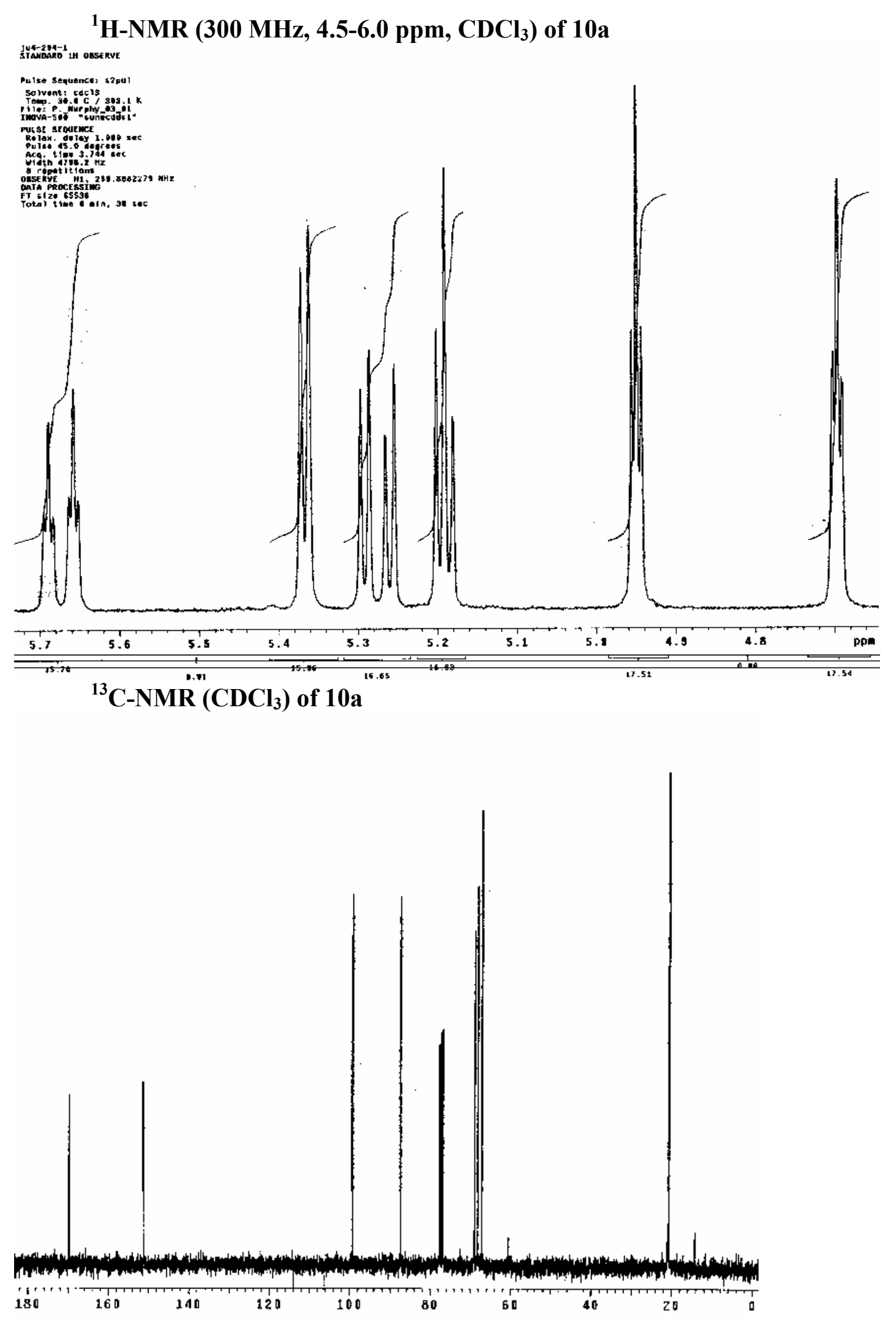


\section{${ }^{1} \mathrm{H}-\mathrm{NMR}\left(300 \mathrm{MHz}, 4.5-6.0 \mathrm{ppm}, \mathrm{CDCl}_{3}\right)$ of 10a}

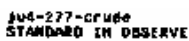

Pulke sequance: arpul

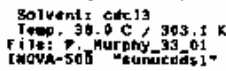

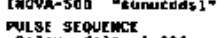

sel las.

Acci:

stritemit tions

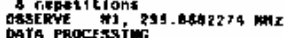

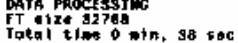

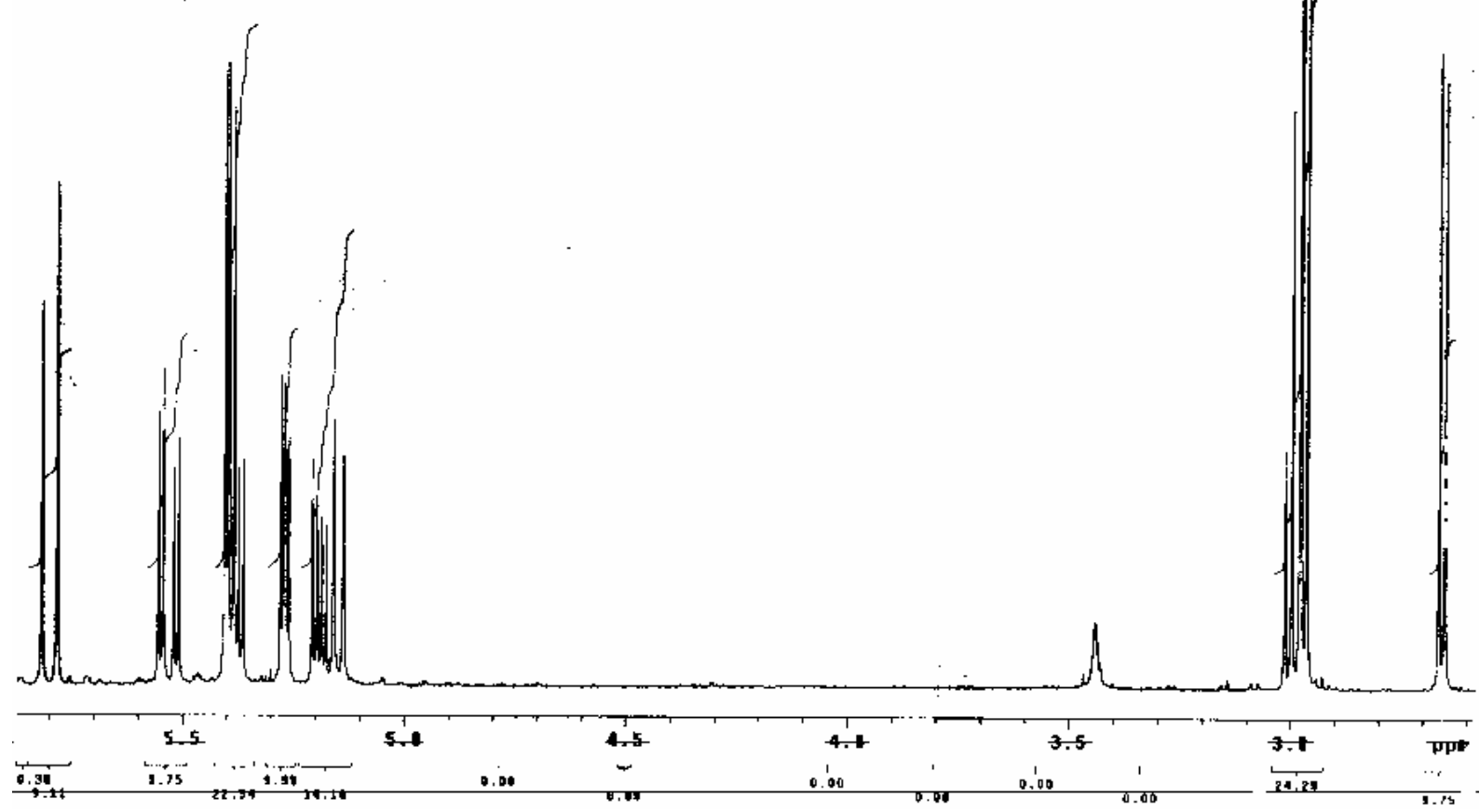


${ }^{1} \mathrm{H}$ NMR (300 MHz, 3-6 ppm, $\left.\mathrm{CDCl}_{3}\right)$ of 13

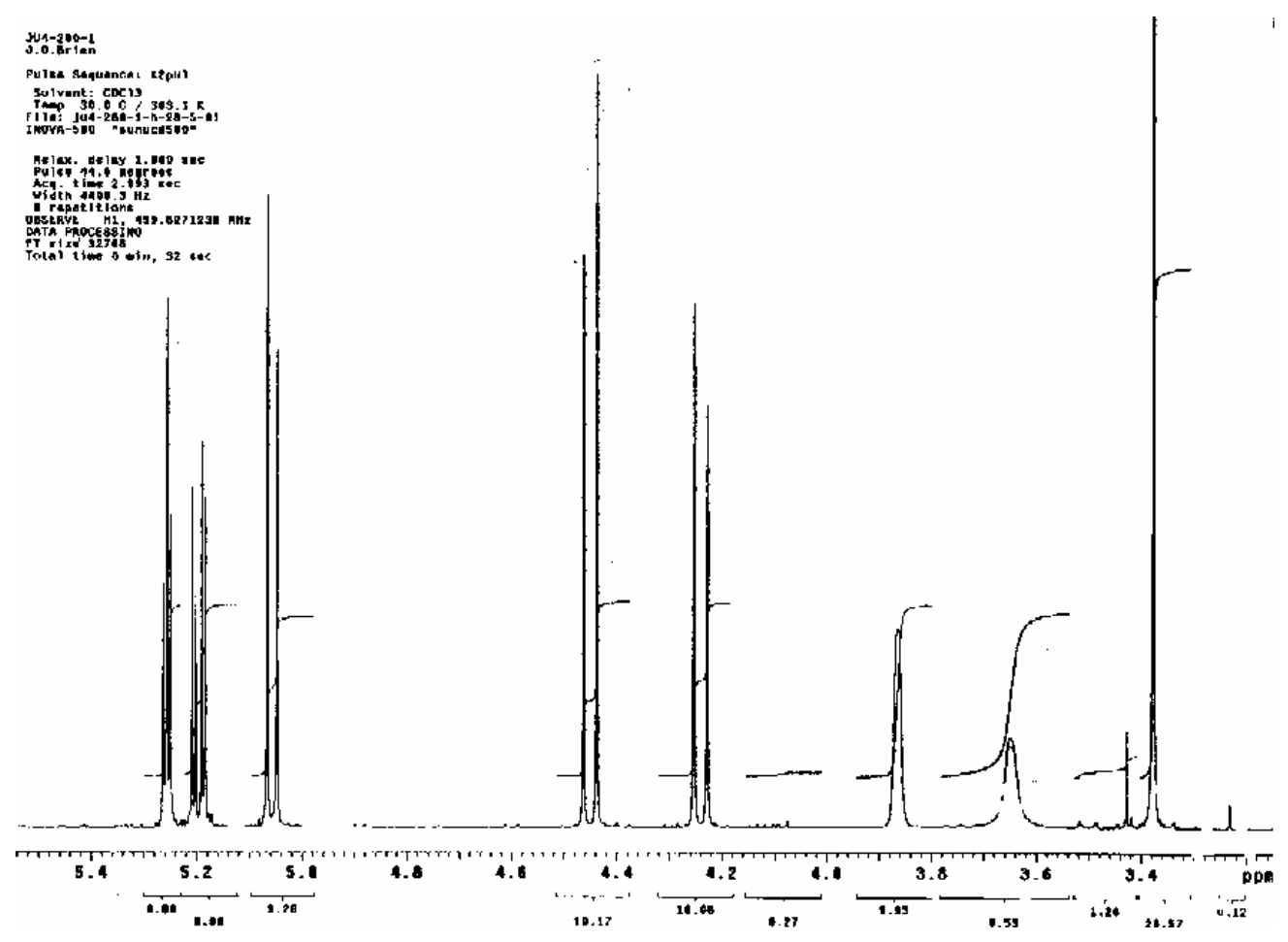

${ }^{13}$ C NMR of $13\left(D_{2} O\right)$

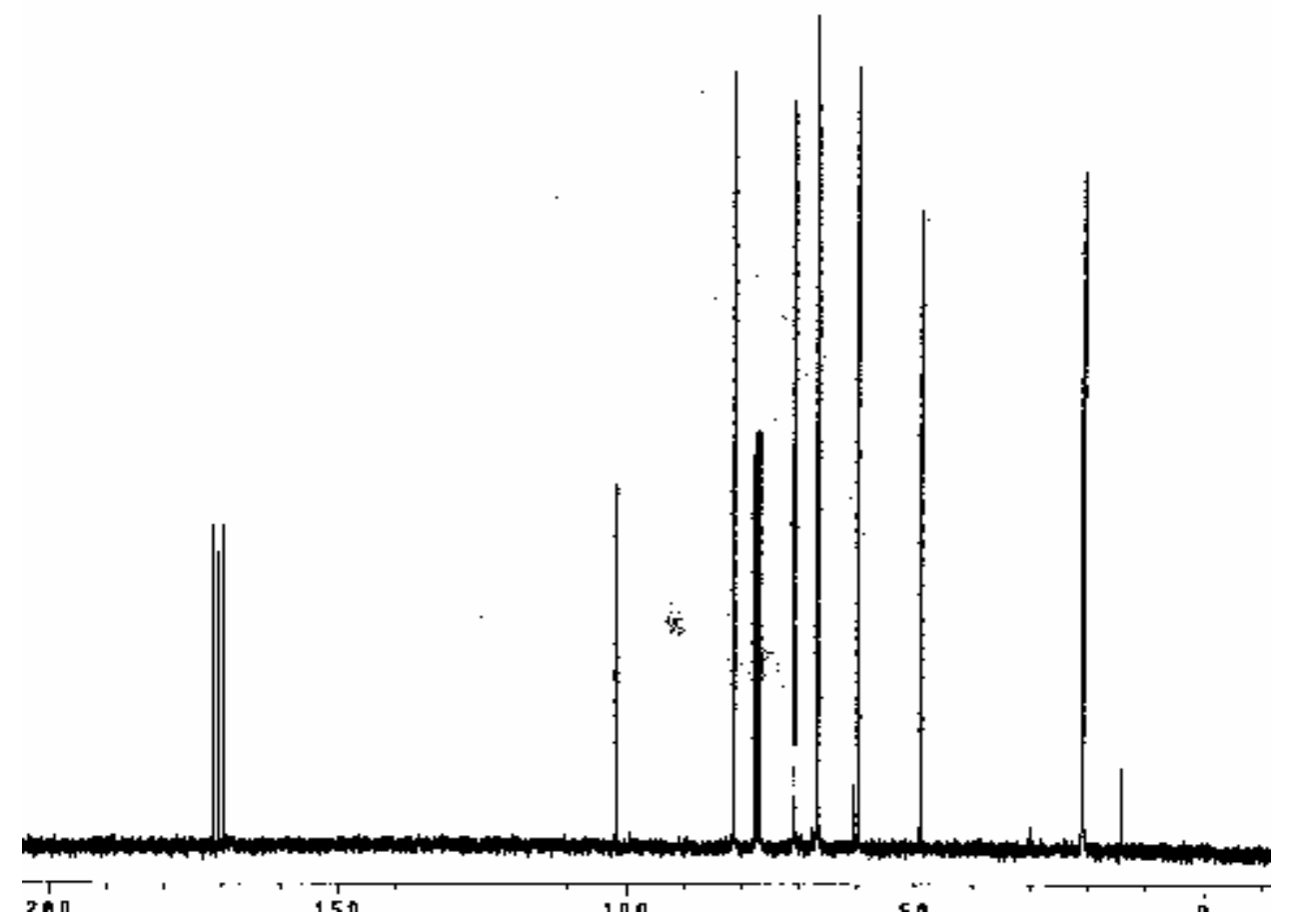


${ }^{1}$ H NMR (300 MHz, D $\left.D_{2} O\right)$ of 14

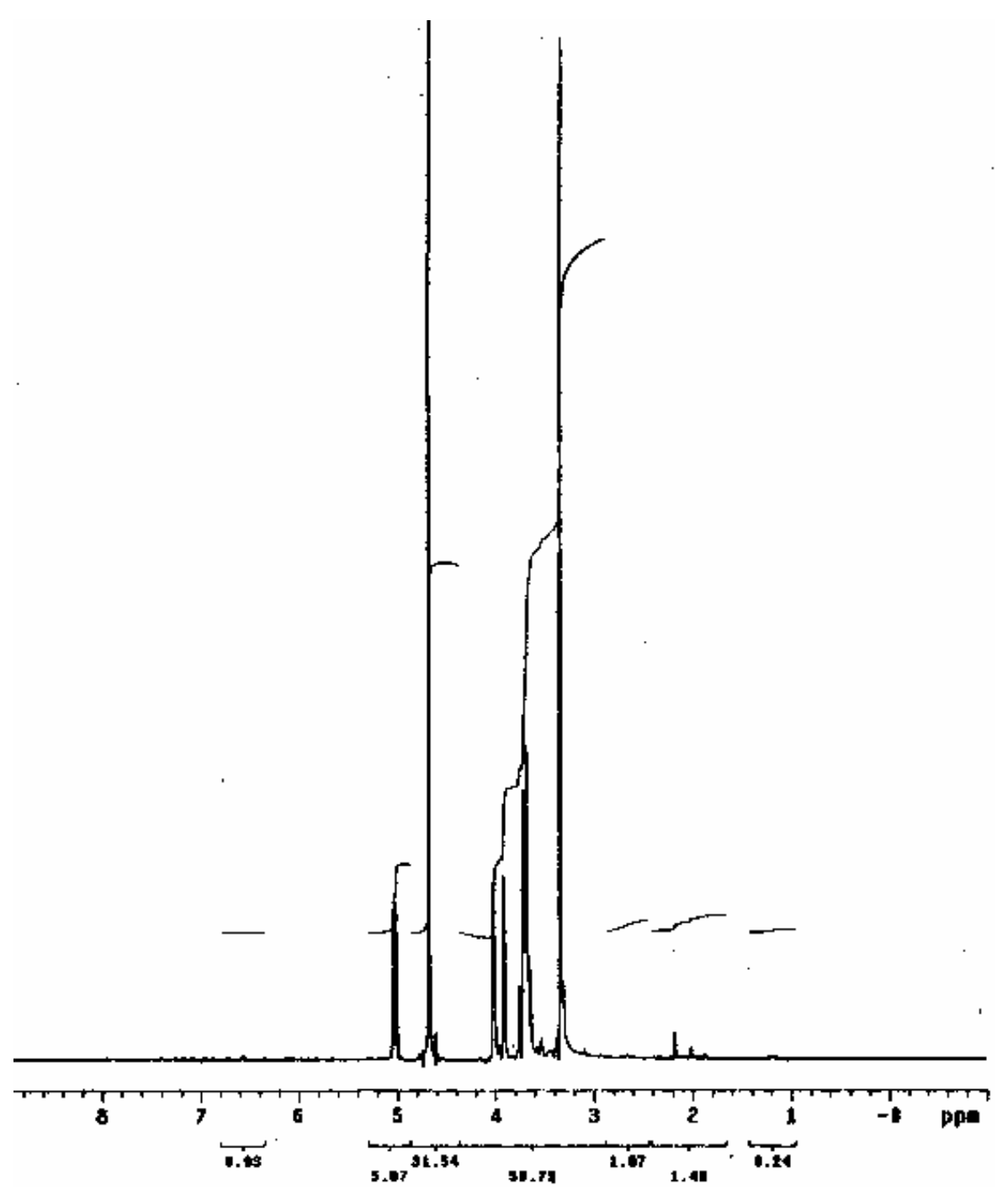

${ }^{13}$ C NMR of $14\left(D_{2} O\right)$

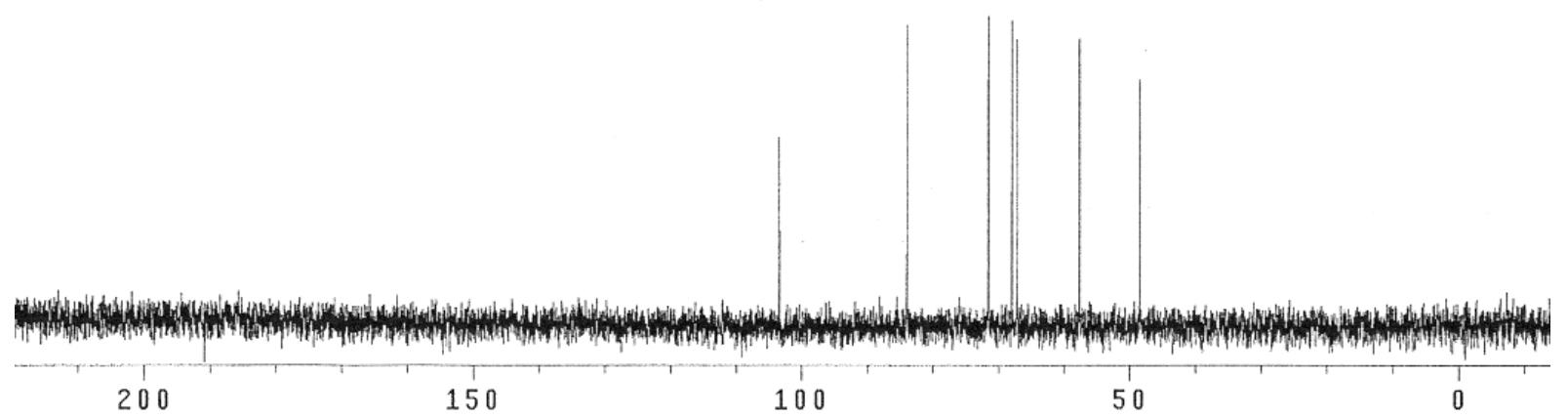




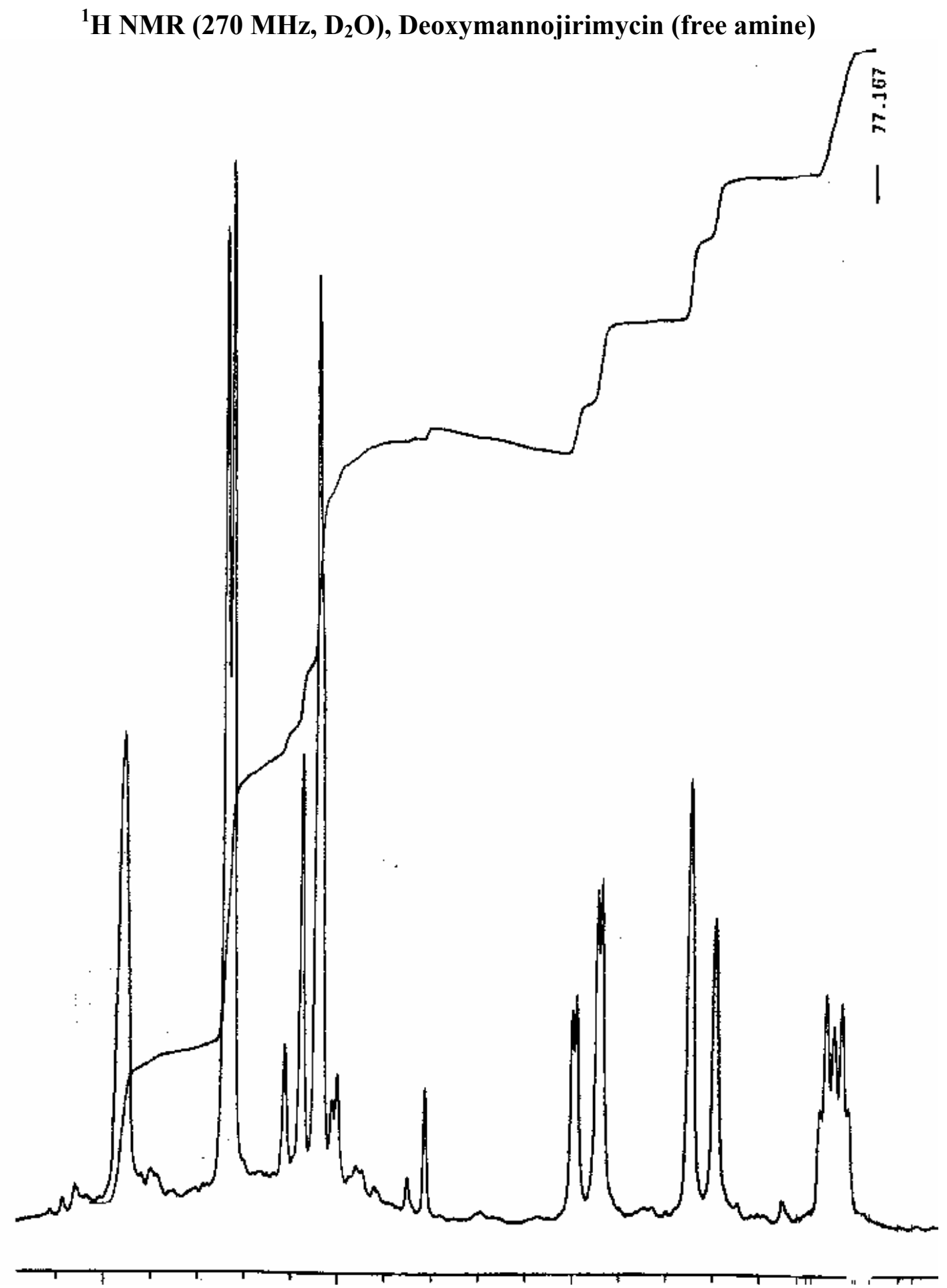




\section{${ }^{1} \mathrm{H}$ NMR (270 MHz, $\mathrm{D}_{2} \mathrm{O}$ ), Deoxymannojirimycin (HCl salt)}

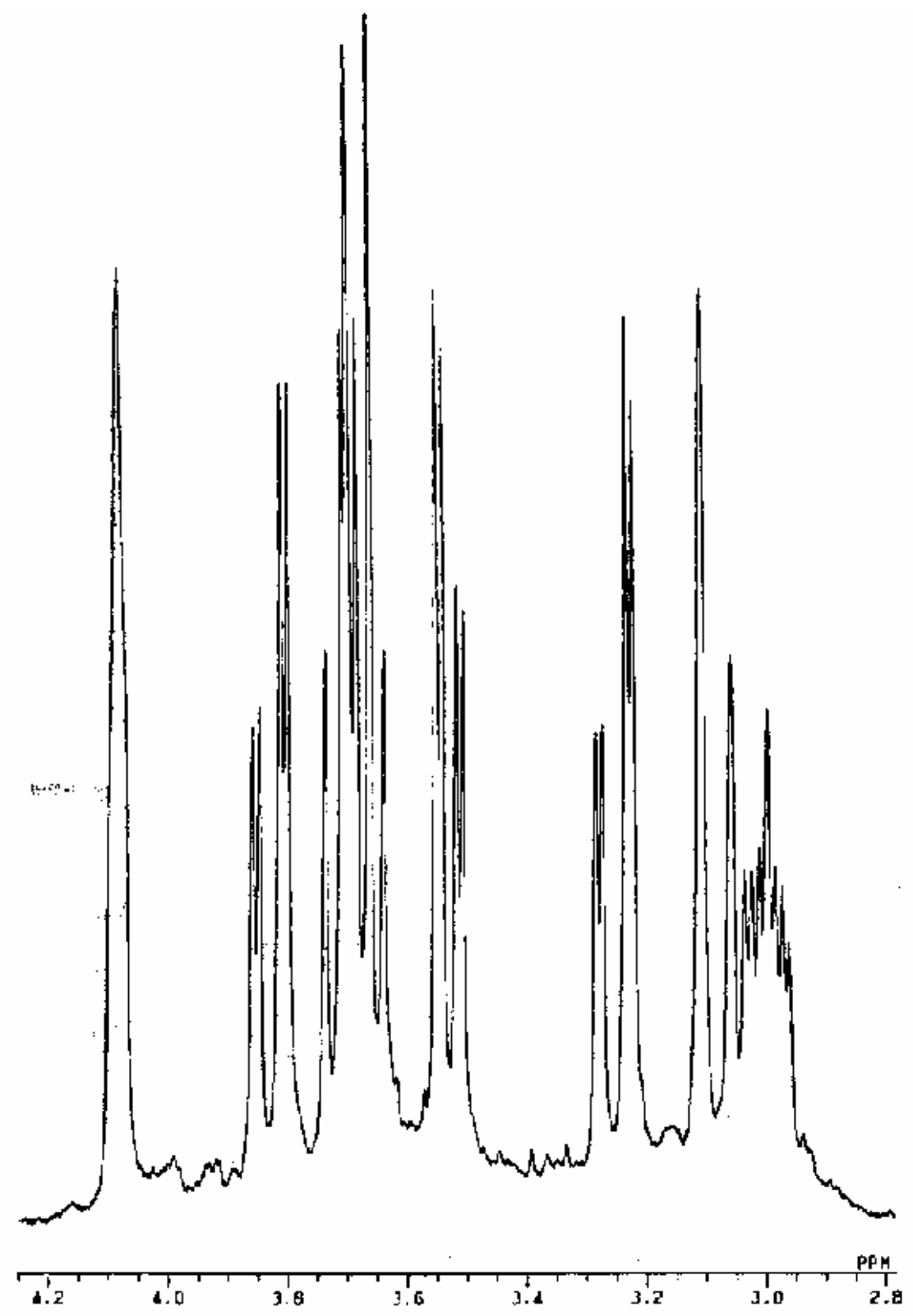




\section{${ }^{1}$ H NMR (270 MHz, D 2 ), Deoxymannojirimycin (crude product unpurified)}

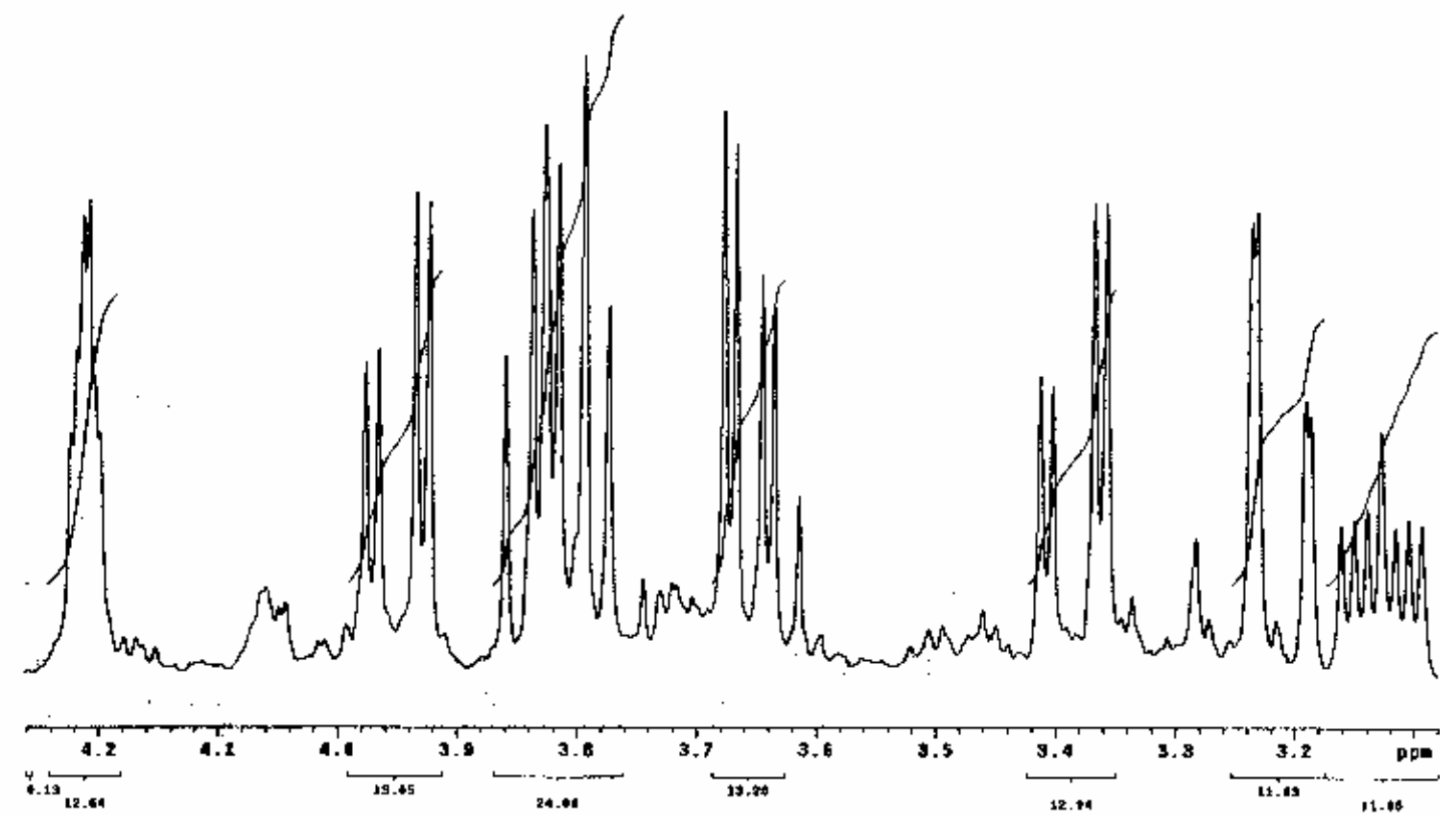




\section{${ }^{1} \mathrm{H}$ NMR (270 MHz, $\left.\mathrm{D}_{2} \mathrm{O}\right)$, 1-Deoxymannojirimycin (HCl salt) from Sigma}

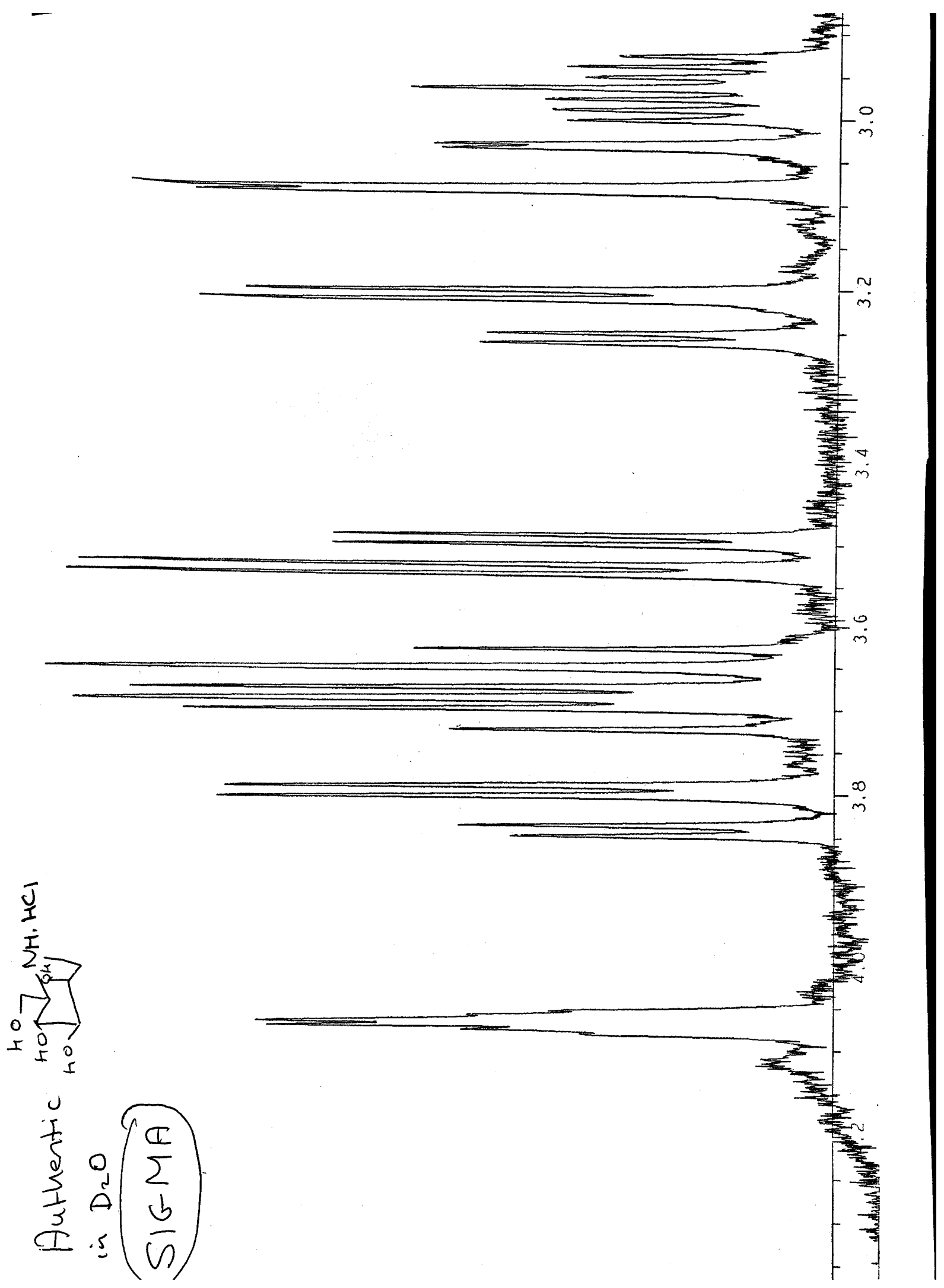


${ }^{1} \mathrm{H}$ NMR (500 MHz, $\mathrm{D}_{2} \mathrm{O}$ ) of synthesized deoxymannojirimycin (HCl salt) 2 after chromatography and salt formation
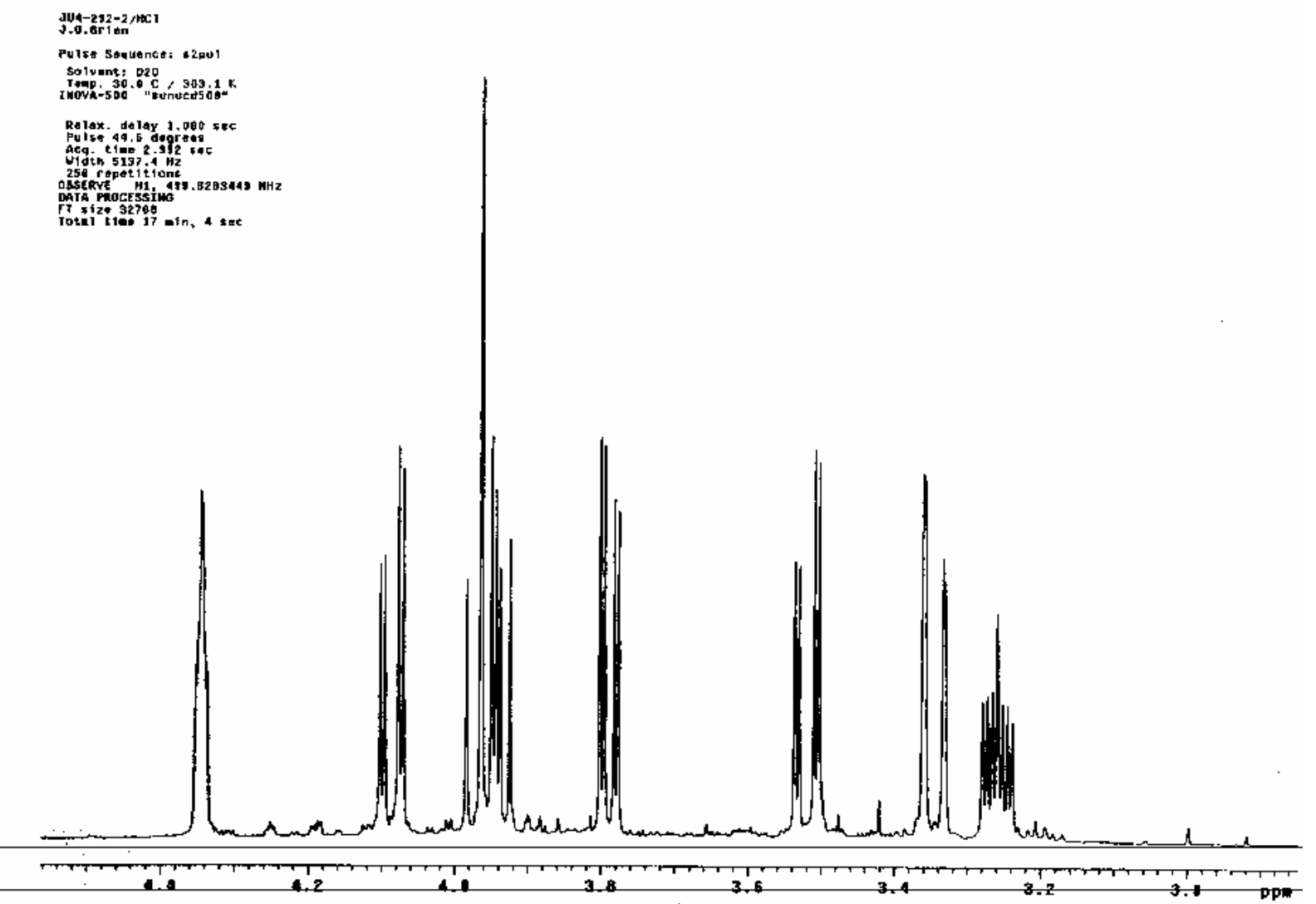
${ }^{1} \mathrm{H}$ NMR (300 MHz, $\mathrm{CDCl}_{3}$ ) of Epoxides 11b

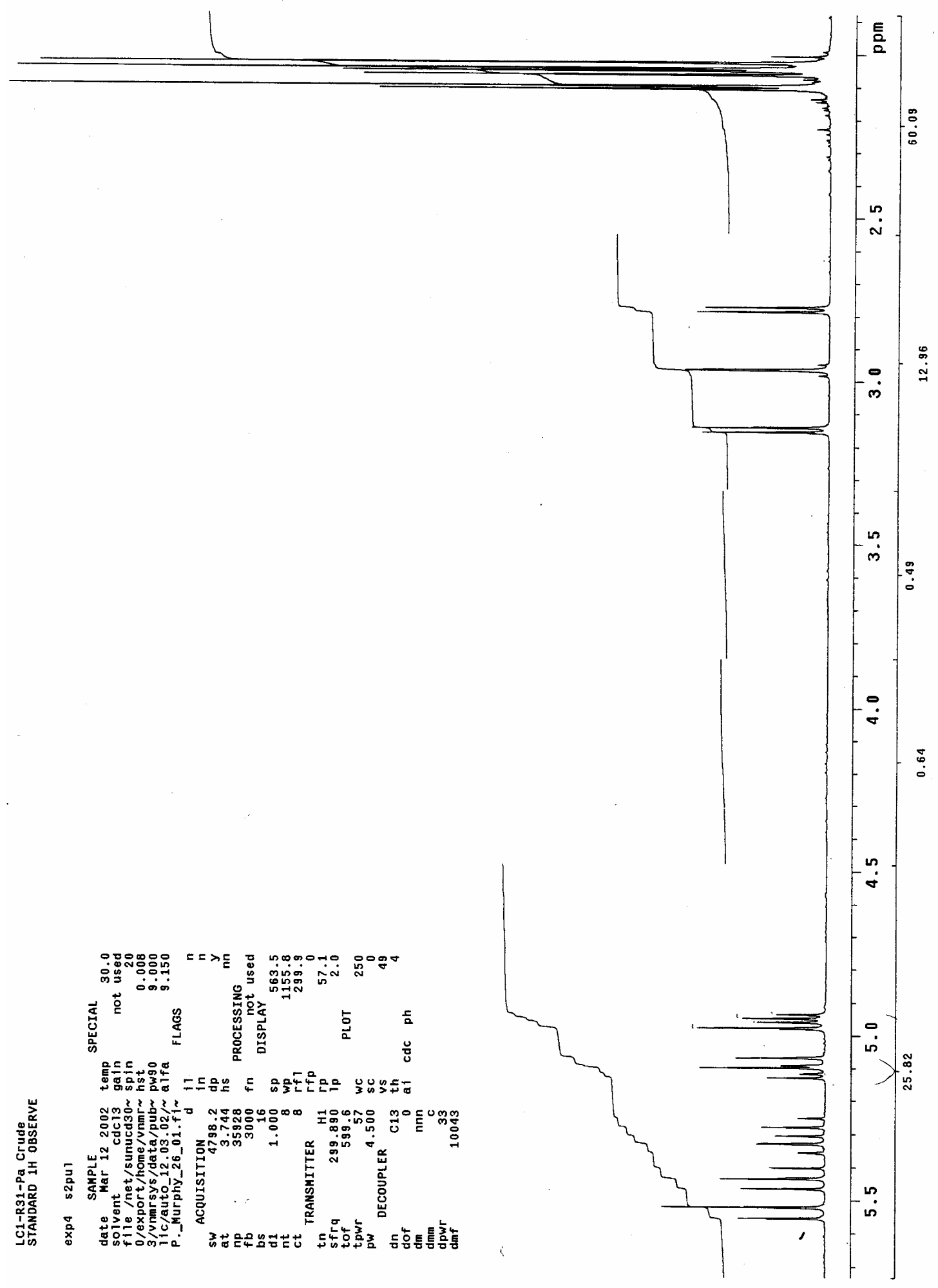




\section{${ }^{13} \mathrm{C}$ NMR (300 MHz, $\left.\mathrm{CDCl}_{3}\right)$ of Epoxides 11b}

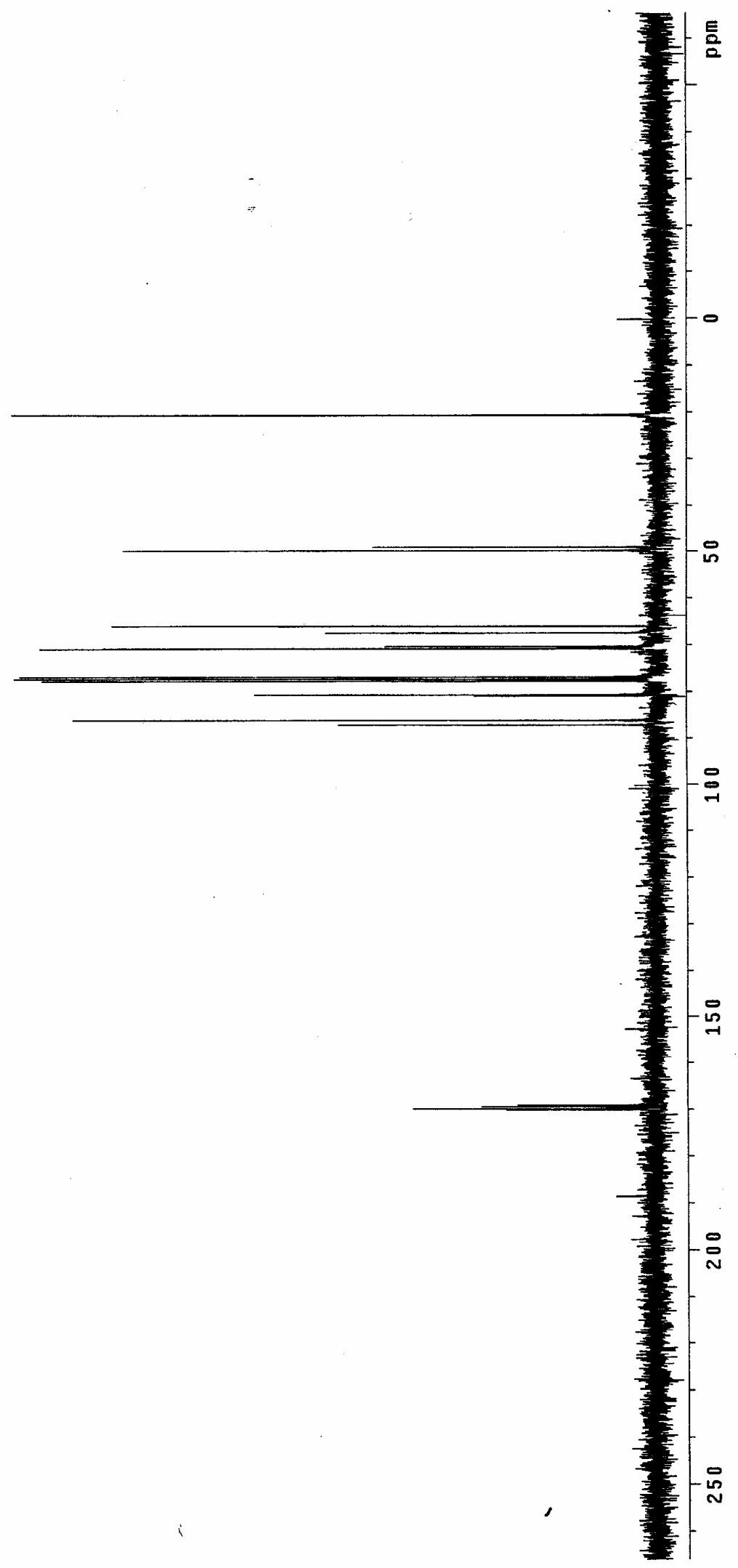




\section{${ }^{1} \mathrm{H}-\mathrm{NMR}\left(300 \mathrm{MHz}, \mathrm{CDCl}_{3}\right)$ of 19}

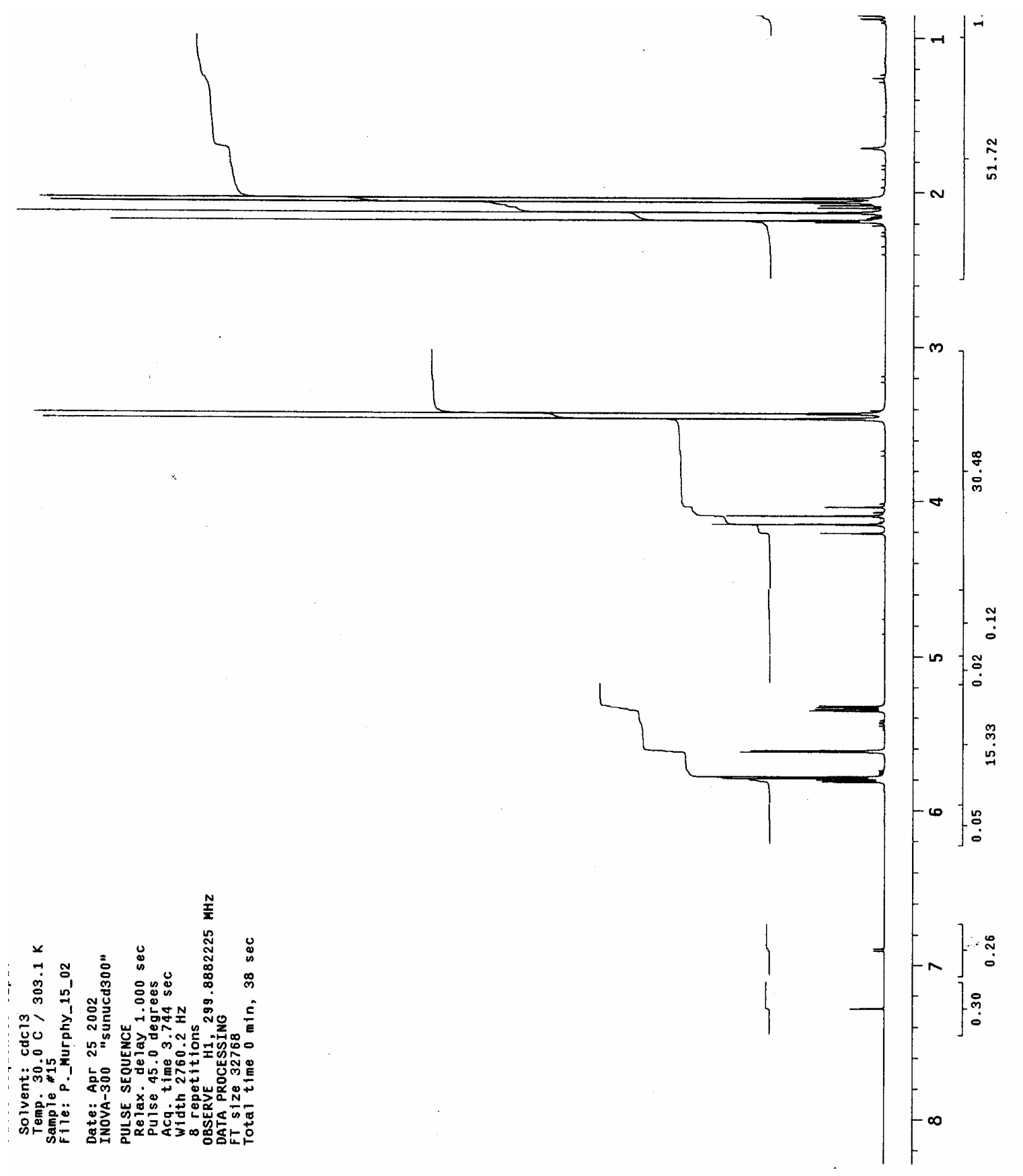




$$
E
$$




\section{${ }^{1} \mathrm{H}-\mathrm{NMR}$ (300 MHz, $\mathrm{CDCl}_{3}$ ) of 12 (Isomer 1)}

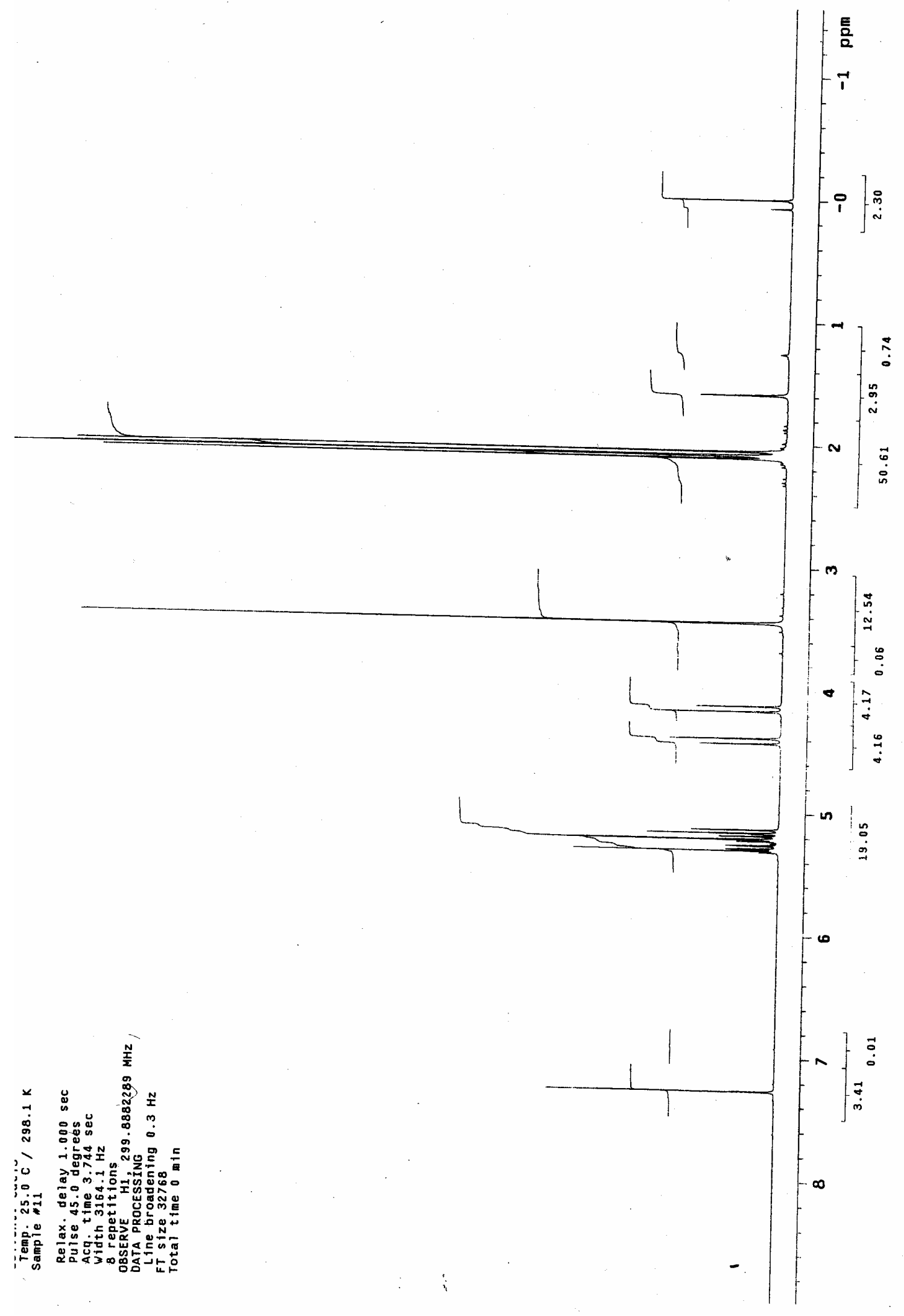




\section{${ }^{1} \mathrm{H}-\mathrm{NMR}\left(300 \mathrm{MHz}, \mathrm{CDCl}_{3}\right.$ ) of 13 (Isomer 2)}

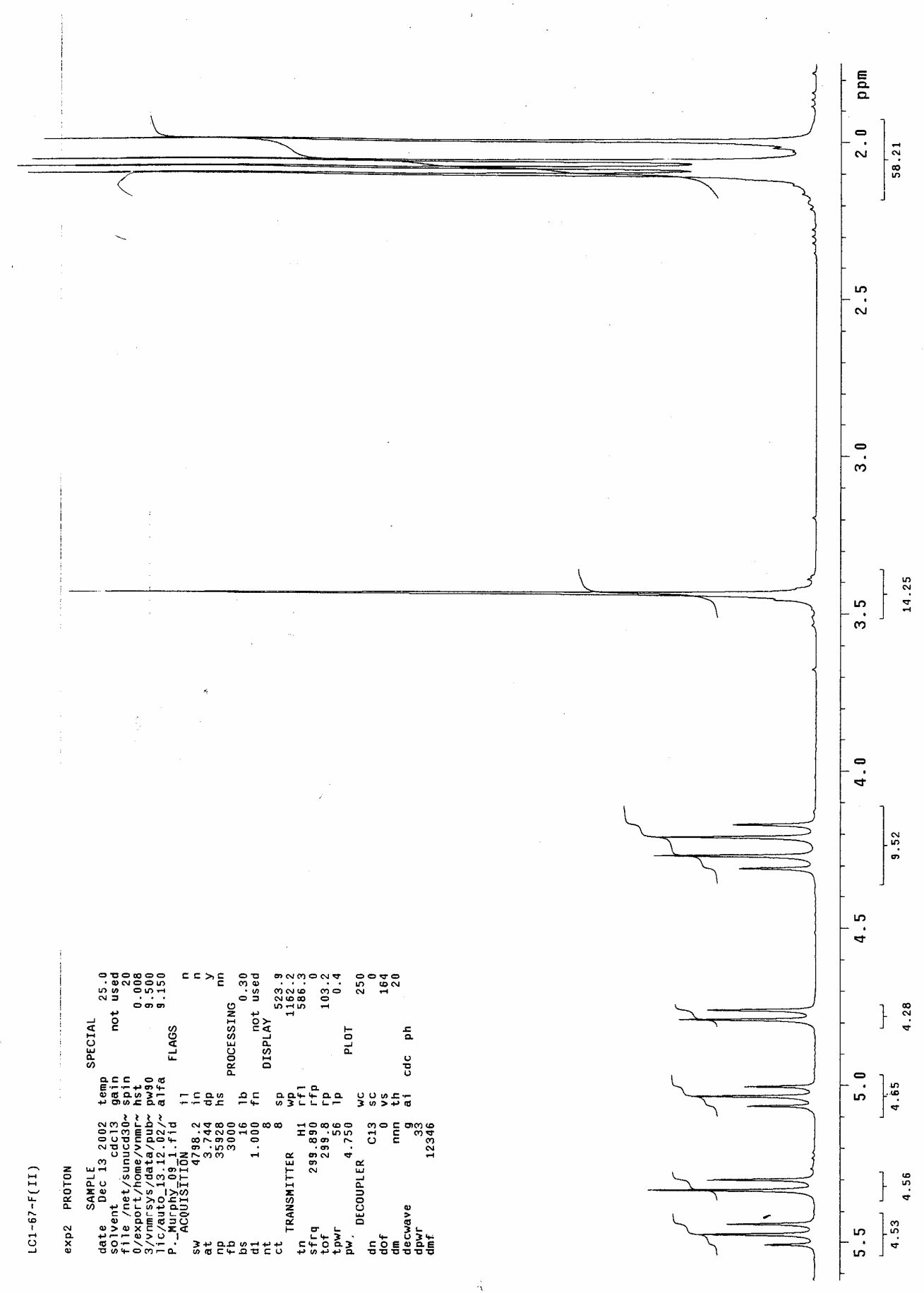




\section{${ }^{1} \mathrm{H}-\mathrm{NMR}\left(\mathrm{D}_{2} \mathrm{O}, 300 \mathrm{MHz}\right)$ 1-Deoxynojirimycin (crude product after hydrogenation)}

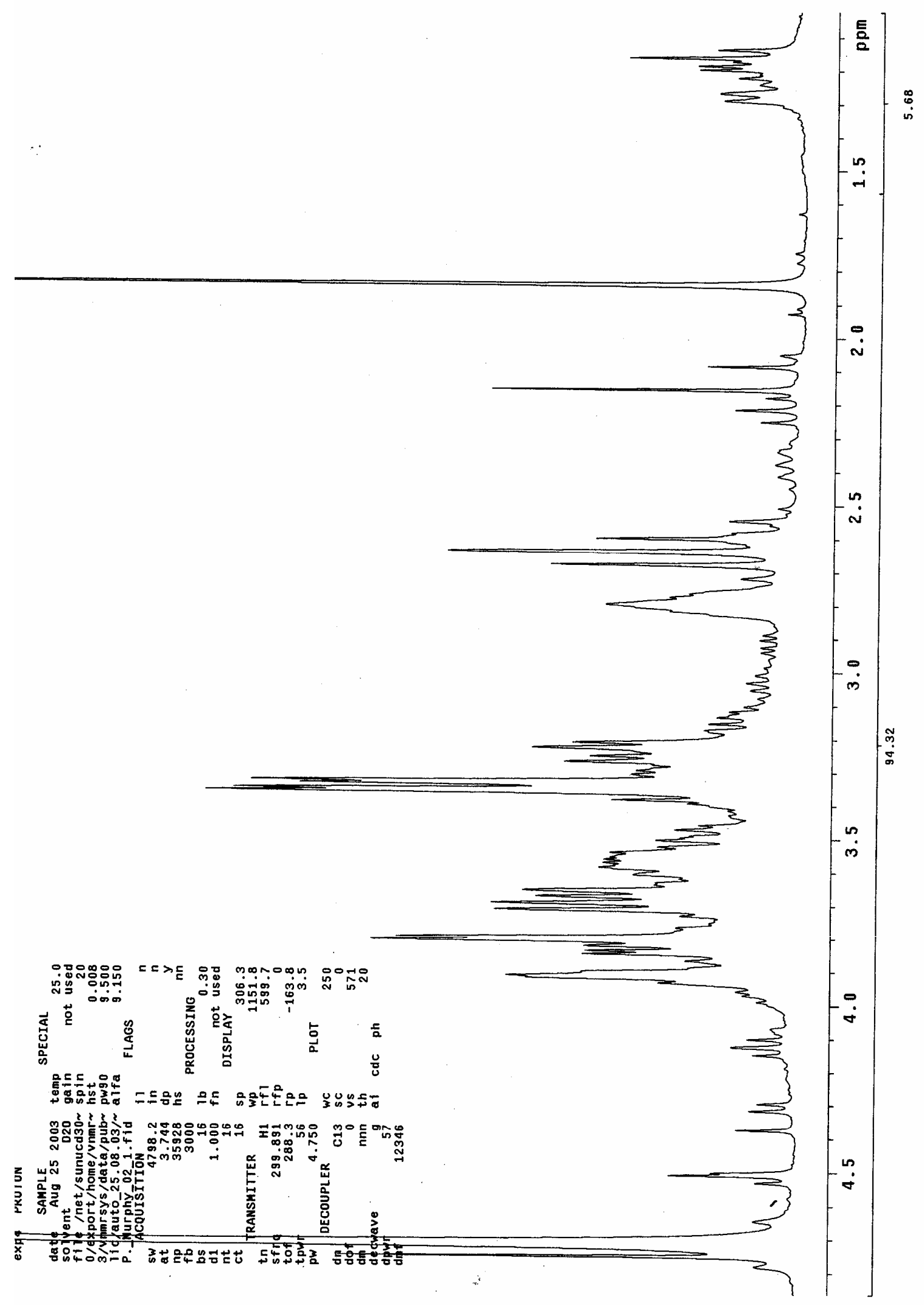




\section{${ }^{1} \mathrm{H}-\mathrm{NMR}\left(\mathrm{D}_{2} \mathrm{O}, 300 \mathrm{MHz}\right)$ 1-Deoxynojirimycin 1 (HCI salt, after purification)}

5
8
6
6
6
6 
${ }^{1} \mathrm{H}-\mathrm{NMR}\left(\mathrm{D}_{2} \mathrm{O}, 300 \mathrm{MHz}\right)$ 1-Deoxynojirimycin (HCI salt, Sigma, authentic sample)

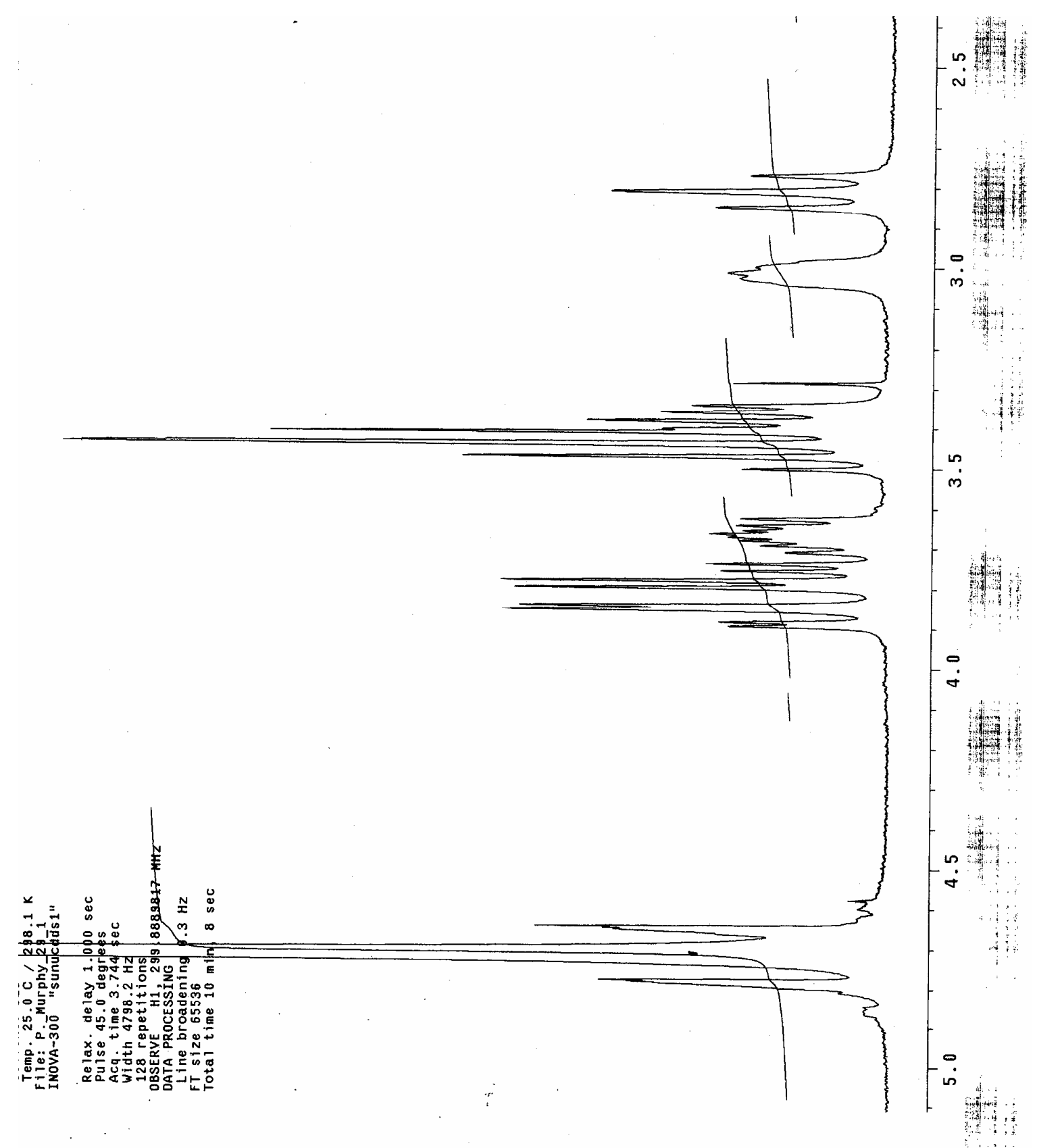




\section{${ }^{1} \mathrm{H}-\mathrm{NMR}\left(\mathrm{D}_{2} \mathrm{O}, 300 \mathrm{MHz}\right)$ 1-Deoxynojirimycin (amine, after chromatography)}

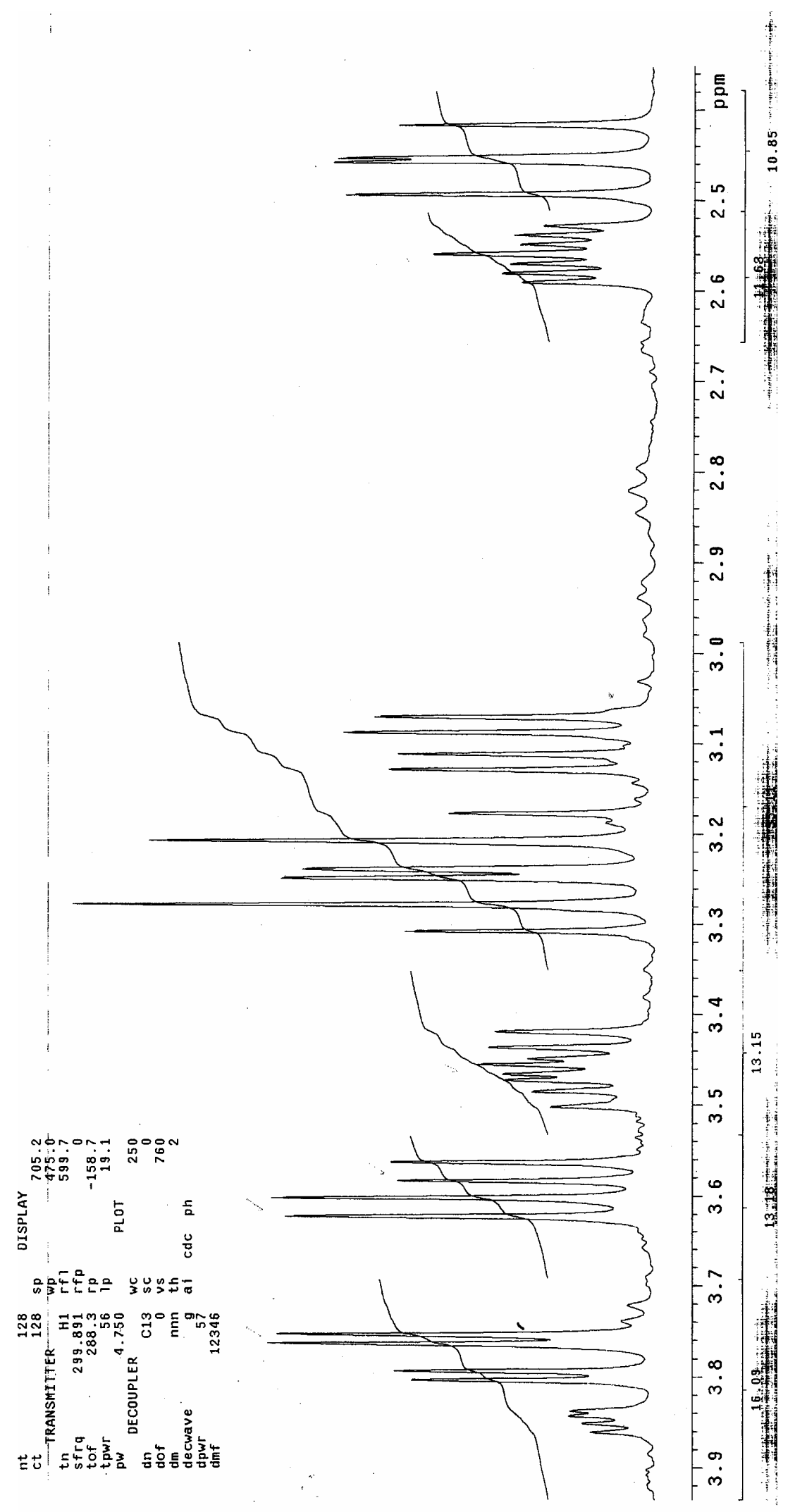


${ }^{13} \mathrm{C}-\mathrm{NMR}\left(300 \mathrm{MHz}, \mathrm{CDCl}_{3}\right.$ ) of 22 (one disastereoisomer)

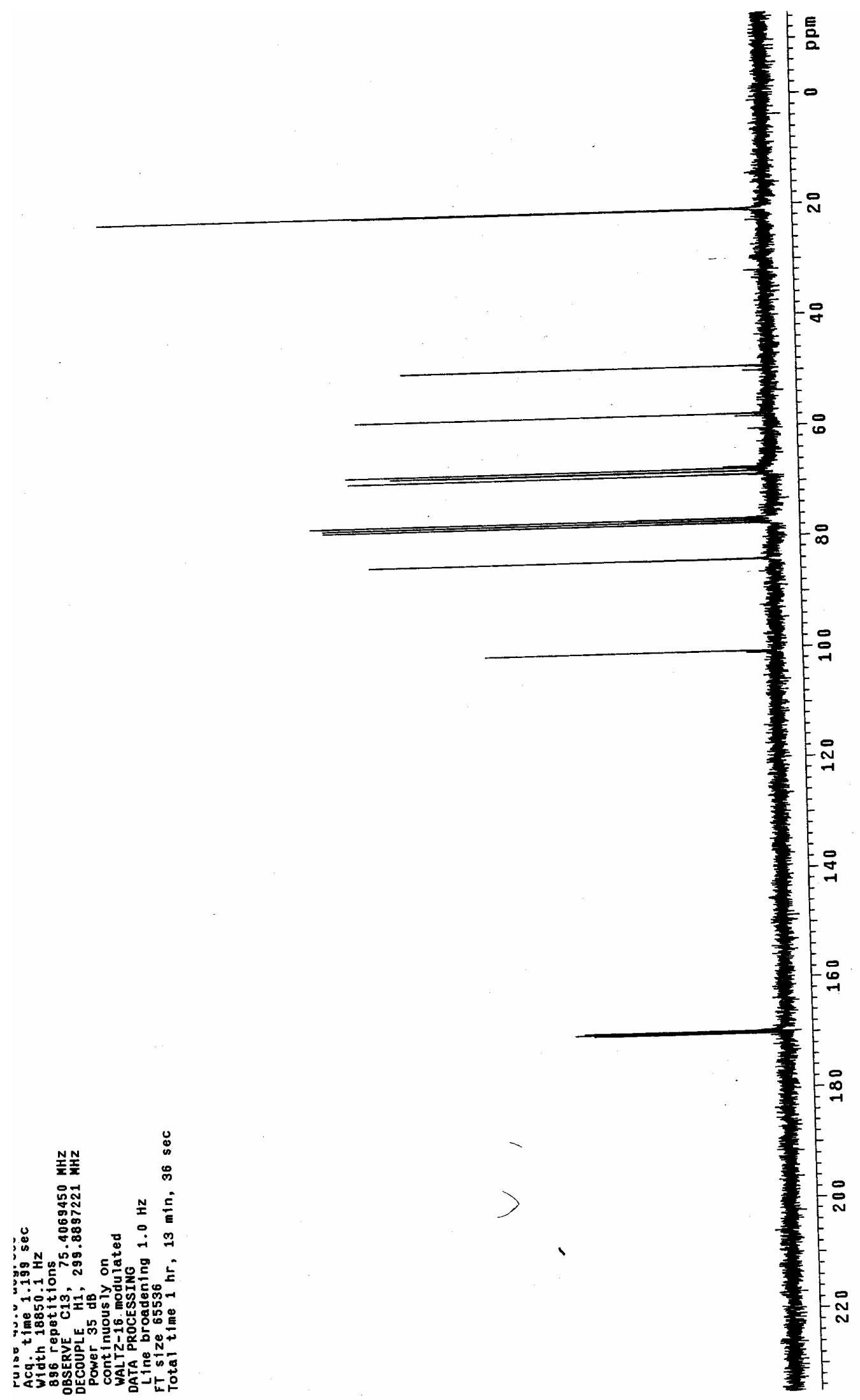


${ }^{1} \mathrm{H}-\mathrm{NMR}\left(300 \mathrm{MHz}, \mathrm{CDCl}_{3}\right.$ ) of 23 (one disastereoisomer)

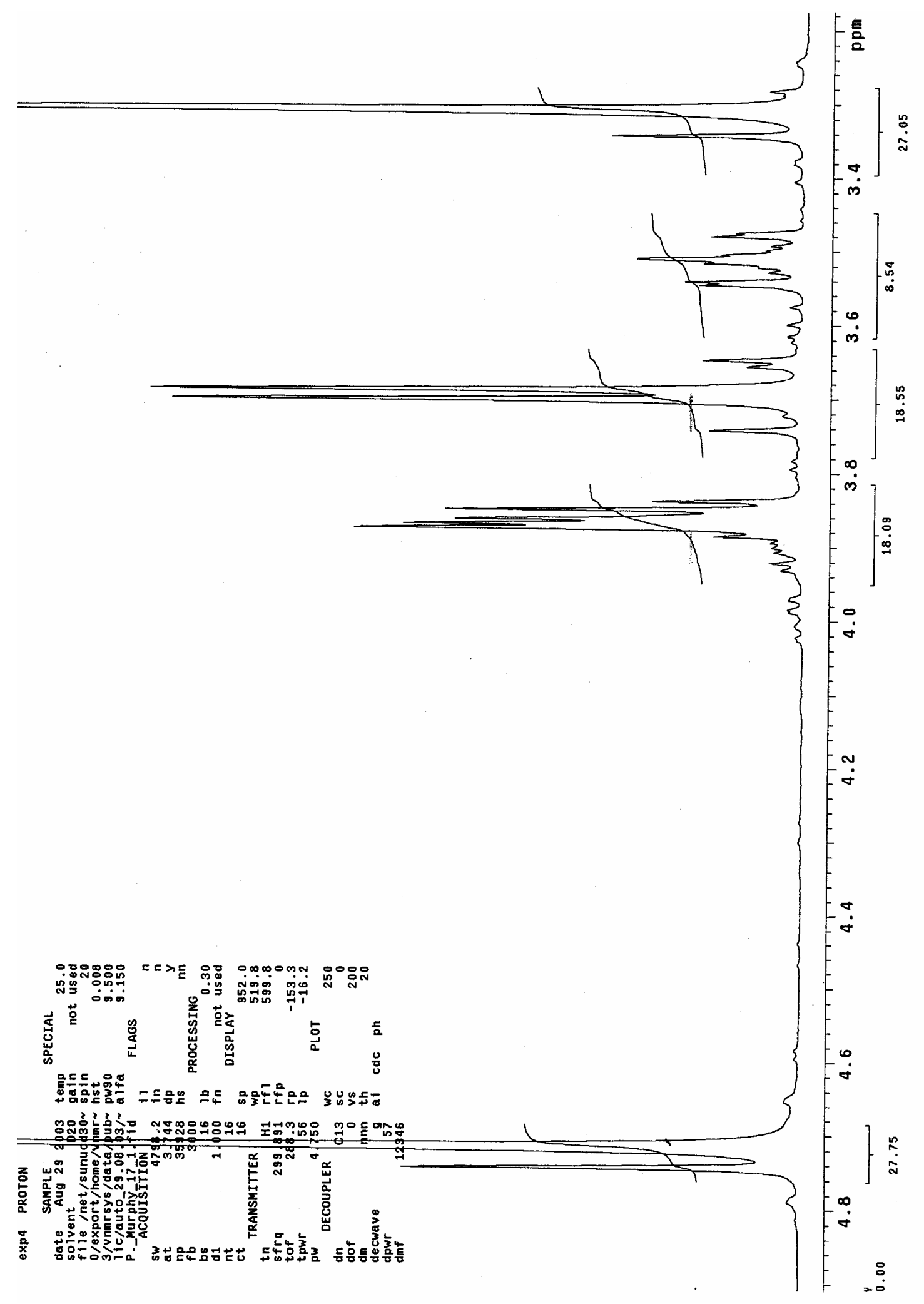




\section{${ }^{13} \mathrm{C}-\mathrm{NMR}\left(300 \mathrm{MHz}, \mathrm{CDCl}_{3}\right.$ ) of acetylated 21 (one disastereoisomer)}

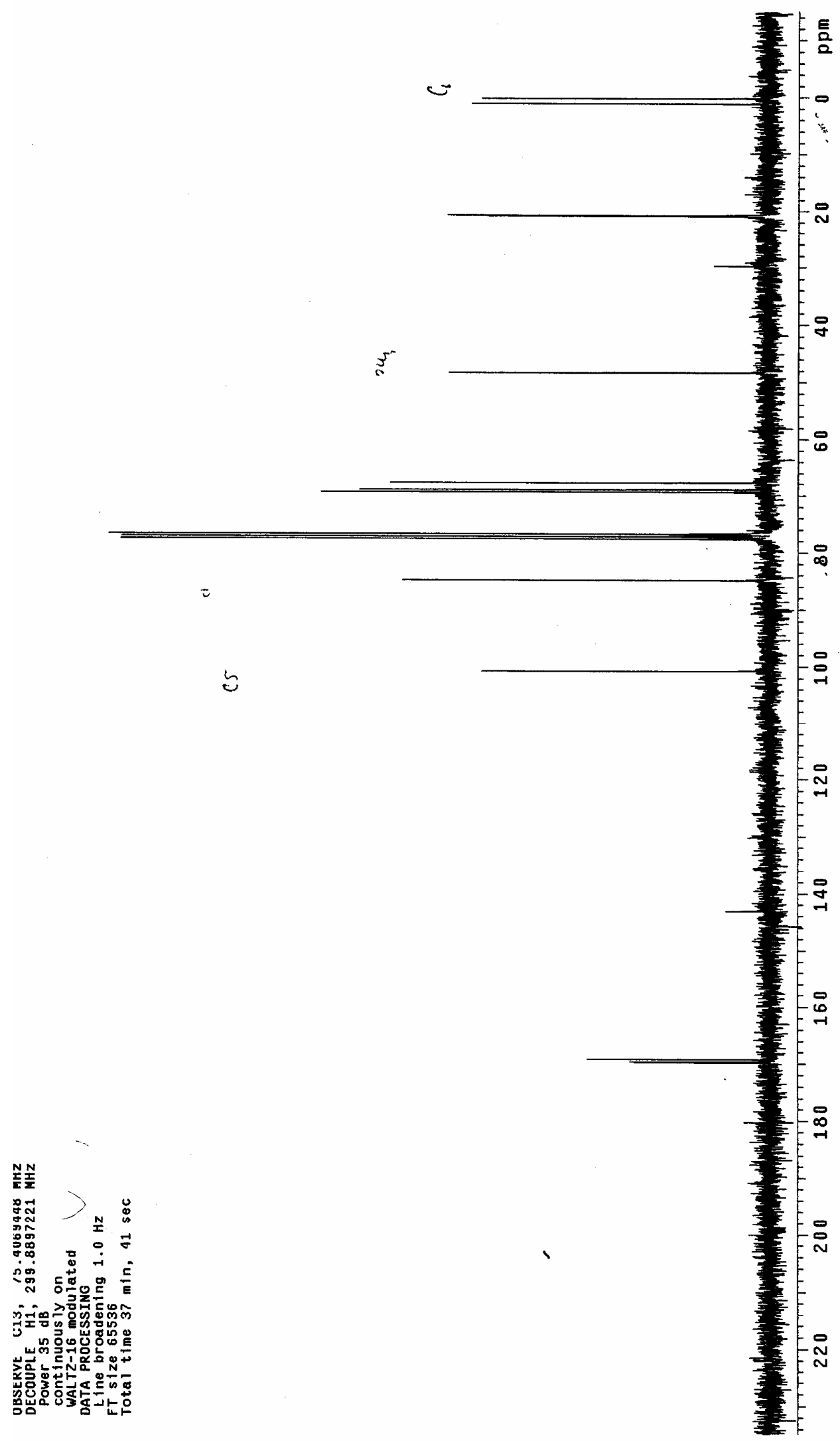




\section{${ }^{1} \mathrm{H}-\mathrm{NMR}\left(\mathrm{D}_{2} \mathrm{O}, 300 \mathrm{MHz}\right)$ 1-Deoxygalactostatin 3 and 1-Deoxyaltronojirimycin 25 (4:1 mixture)}

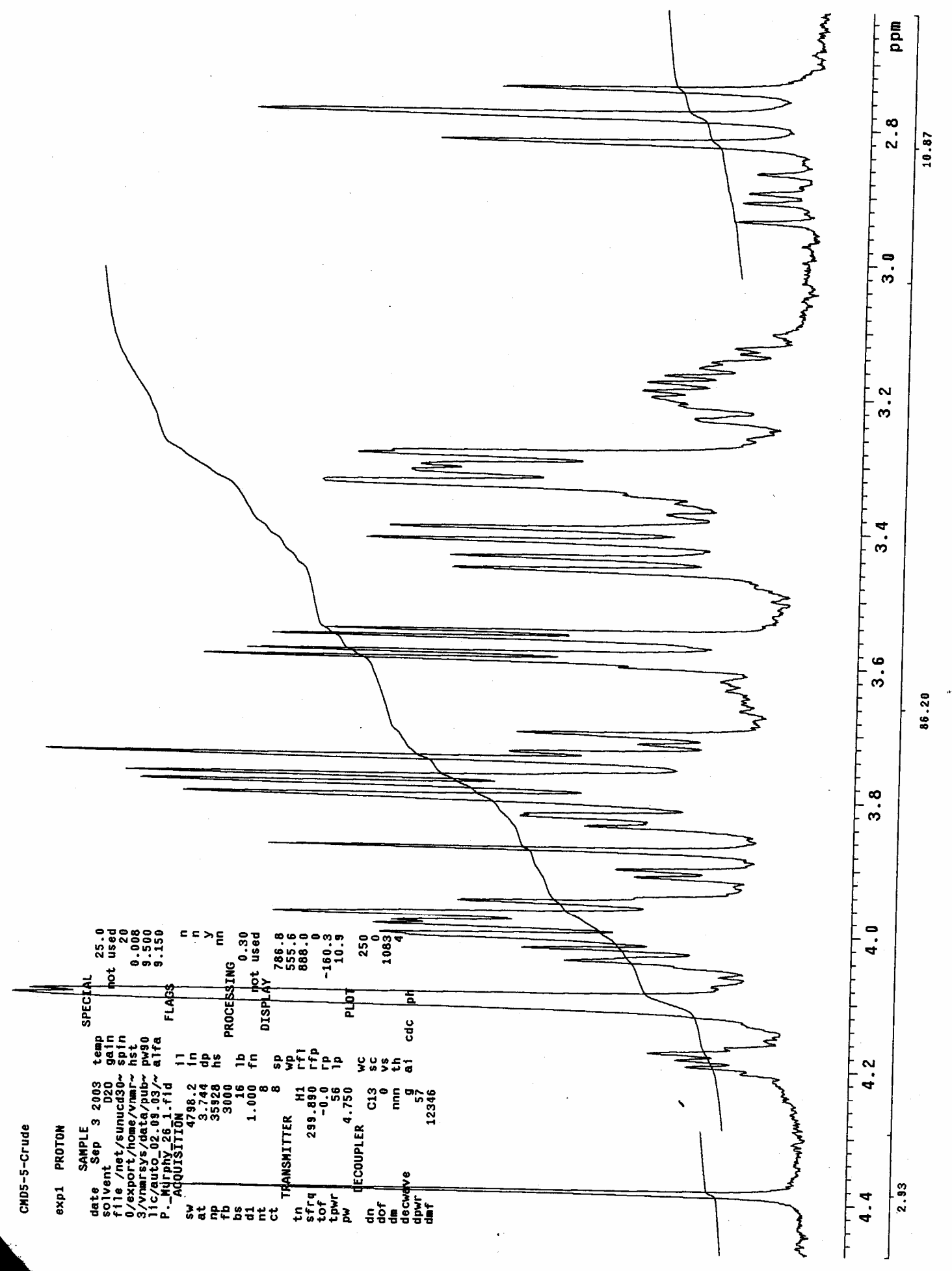




\section{${ }^{1} \mathrm{H}-\mathrm{NMR}\left(\mathrm{D}_{2} \mathrm{O}, 300 \mathrm{MHz}\right)$ 1-Deoxy-D-galactostatin 3 (authentic sample)}

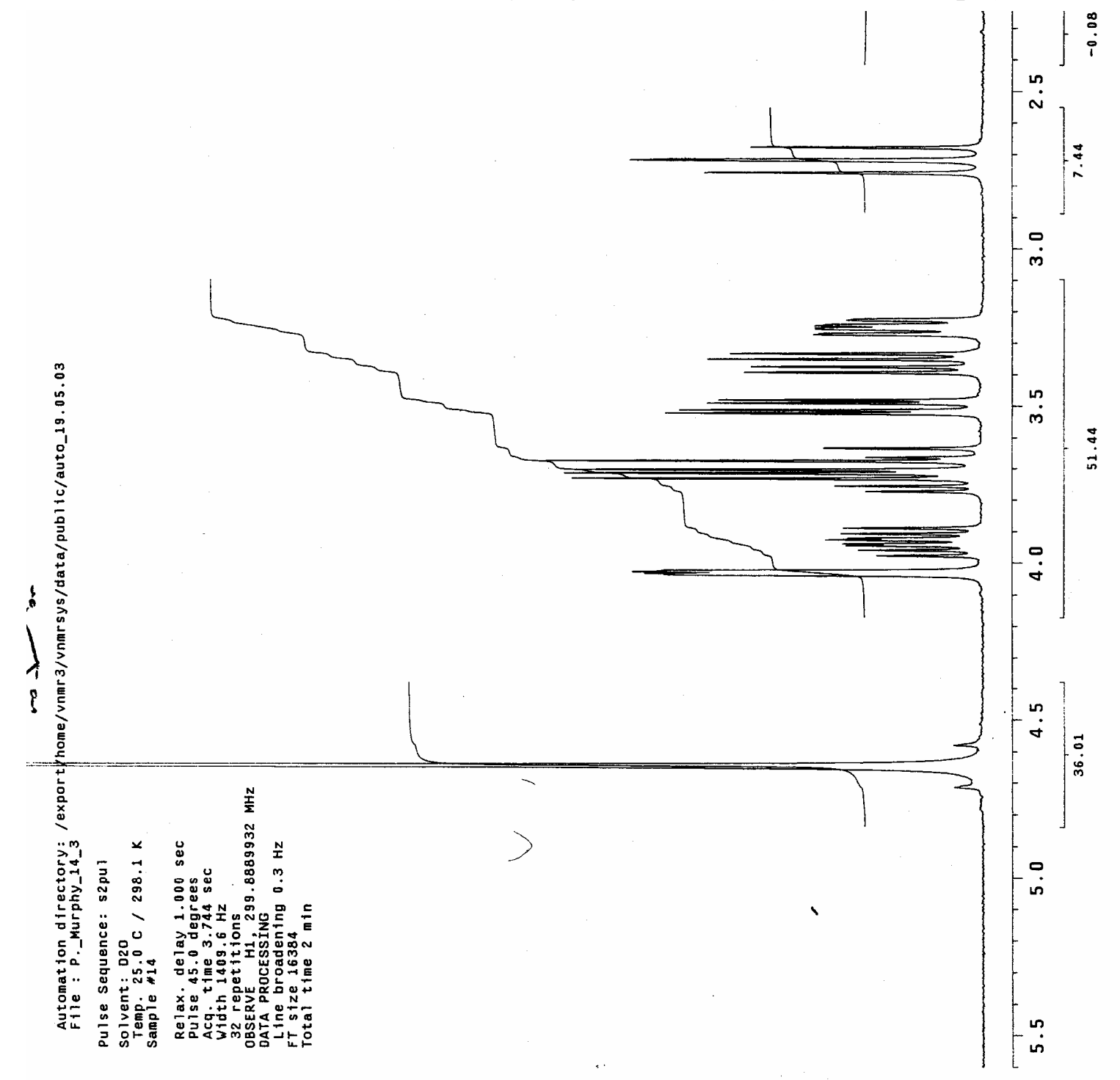




\section{${ }^{1} \mathrm{H}-\mathrm{NMR}\left(\mathrm{D}_{2} \mathrm{O}, 300 \mathrm{MHz}\right.$ ) 1,6-dideoxy-D-galactonojirimycin 4 (HCl salt)}

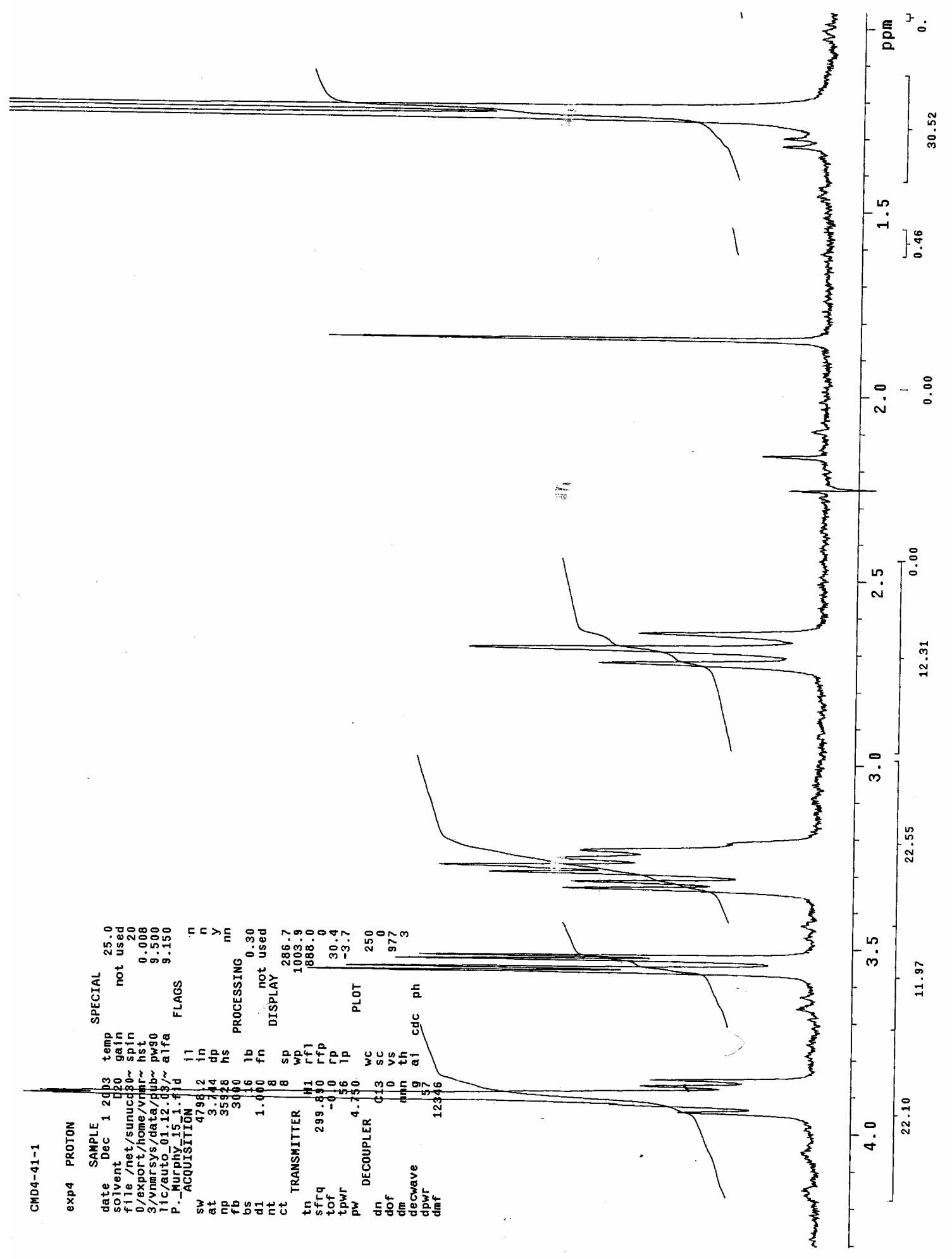


${ }^{1} \mathrm{H}-\mathrm{NMR}\left(\mathrm{D}_{2} \mathrm{O}, 300 \mathrm{MHz}\right)$ 1-Deoxy-L-fuconojirimycin (enantiomer of 4, HCl salt) authentic sample

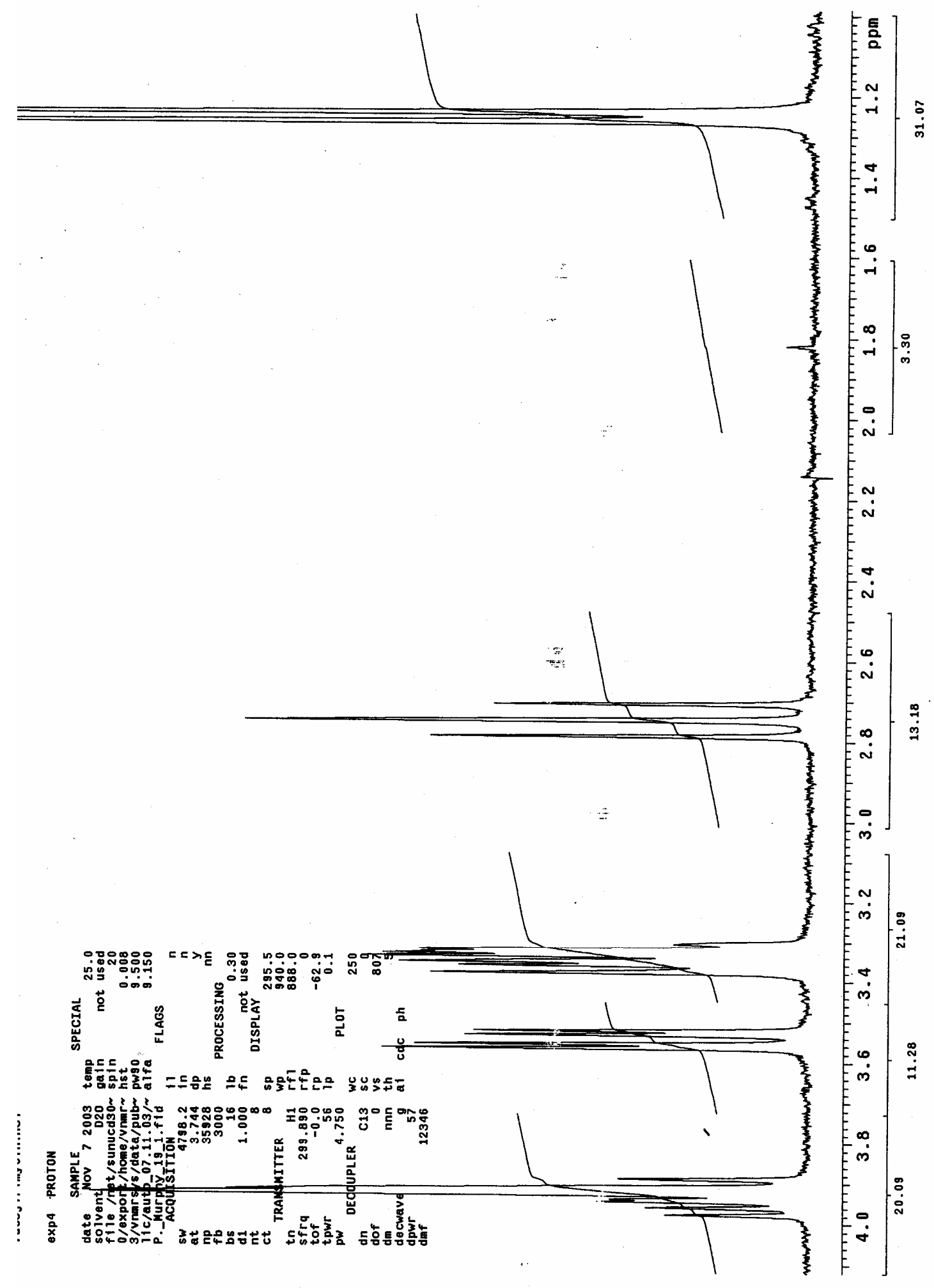

